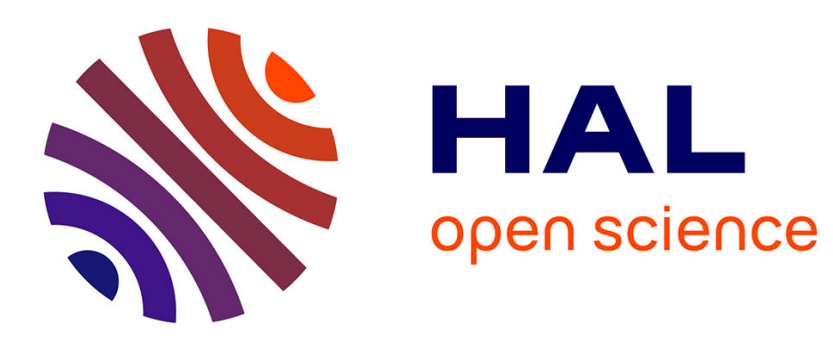

\title{
Impact du changement climatique sur l'agriculture : une quantification du biais de prix dans les approches économétriques
}

\author{
Fabrice Ochou, Philippe Quirion
}

\section{- To cite this version:}

Fabrice Ochou, Philippe Quirion. Impact du changement climatique sur l'agriculture: une quantification du biais de prix dans les approches économétriques. Revue Economique, A paraître, Prépublication (7), pp.I-XXV. 10.3917/reco.731.0043 . hal-03499241

\section{HAL Id: hal-03499241 \\ https://hal.science/hal-03499241}

Submitted on 21 Dec 2021

HAL is a multi-disciplinary open access archive for the deposit and dissemination of scientific research documents, whether they are published or not. The documents may come from teaching and research institutions in France or abroad, or from public or private research centers.
L'archive ouverte pluridisciplinaire HAL, est destinée au dépôt et à la diffusion de documents scientifiques de niveau recherche, publiés ou non, émanant des établissements d'enseignement et de recherche français ou étrangers, des laboratoires publics ou privés. 


\section{Impact du changement climatique sur l'agriculture : une quantification du biais de prix dans les approches économétriques Fabrice OCHOU, Philippe QUIRION}

Beaucoup d'études économétriques évaluent l'impact du changement climatique sur l'agriculture en supposant les prix agricoles exogènes. Si les variables climatiques influencent ces prix, il en résulte un «biais de prix », dont l'existence est reconnue mais qui n'a jamais été quantifié. Nous quantifions ce biais dans le cas du Burkina Faso, avec un panel de 45 provinces et 14 années, pour les trois céréales les plus cultivées : maïs, mil et sorgho.

Le rendement en grain est sensible aux chocs météorologiques. Si ces chocs diminuent le rendement en grain de $10 \%$, le prix des céréales augmente de 2 à $3 \%$. Le biais de prix est important, puisque la perte de bien-être est $20 \%$ à $70 \%$ plus élevée que celle calculée en supposant les prix exogènes. L'ampleur du biais dépend de celle du choc météorologique, du modèle économétrique utilisé et des hypothèses retenues sur les coûts de production.

Many econometric studies assess the impact of climate change on agriculture by assuming exogenous agricultural prices. If climate variables influence these prices, the result is a 'price bias', the existence of which is recognised but has never been quantified. We quantify this bias in the case of Burkina Faso, with a panel of 45 provinces and 14 years for the three most widely grown cereals: maize, millet and sorghum.

Grain yields are sensitive to weather shocks. If these shocks reduce grain yield by $10 \%$, the price of grain increases by $2-3 \%$. The price bias is significant, since the welfare loss is $20 \%$ to $70 \%$ higher than that calculated by assuming exogenous prices. The magnitude of the bias depends on the magnitude of the weather shock, the econometric model used and the assumptions made about production costs. 
Mots Clés : Biais de Prix, Changements climatiques, Agriculture.

Keywords: Price bias, climate change, agriculture.

Codes JEL : O13, Q51, Q54

Fabrice Ochou Esse, CIRED, 25 BP 302 Cidex 01 Abidjan 25, Côte d'Ivoire, esse.ochou@gmail.com.

Philippe QUIRION, CIRED, CNRS, 45 bis avenue de la belle Gabrielle, 94736 Nogent-surMarne cedex, quirion@ centre-cired.fr

Remerciements. Pour leurs conseils avisés, nous tenons à remercier deux relecteurs anonymes de la Revue économique, un relecteur anonyme de la série des documents de travail de la FAERE, les participants à la conférence annuelle de la FAERE et au séminaire du CIRED où une version précédente de cet article a été présentée, ainsi que Thierry Kamionka et l'ensemble du Comité de Rédaction de la Revue économique. 


\section{INTRODUCTION}

Les études empiriques consacrées à l'impact du changement climatique sur l'agriculture sont très nombreuses et diverses typologies ont été proposées pour les regrouper; cf. par exemple le dossier sur ce sujet publié dans la Review of Environmental Economics and Policy (Blanc et Reilly, [2017]). L'une des distinctions principales porte sur le choix des variables agricoles, c'est-à-dire celles sur lesquelles on cherche à quantifier l'impact du changement climatique. Une partie de ces travaux porte sur des variables biophysiques, comme la production ou le rendement mesuré en unités physiques (typiquement, pour le rendement, en $\mathrm{kg}$ de grain par hectare) ou encore la biomasse totale. Ces travaux sont le plus souvent menés par des agronomes (Rosenzweig et al., [1993]) ou des chercheurs en science de l'environnement (Tigchelaar et al., [2018]), mais parfois aussi par des économistes (Schlenker et Roberts, 2009). Une autre partie des travaux, généralement menés par des économistes, porte sur des variables exprimées en grandeurs monétaires : valeur de la production agricole, revenu agricole ou valeur des terres. Par concision, nous baptiserons les premières « approches agronomiques » et les secondes « approches économétriques ».

L'article le plus influent au sein de cette deuxième famille est celui de Mendelsohn, Nordhaus et Shaw [1994] - MNS dans la suite du texte - fondateur de l'approche que ces auteurs ont baptisé «ricardienne». MNS mènent des régressions en coupe instantanée expliquant la valeur des terres par des variables climatiques, en l'occurrence les précipitations et la température à différentes périodes de l'année, à l'échelle des Etats-Unis. Cette focalisation sur la valeur des terres se base sur l'idée selon laquelle cette valeur est déterminée par le revenu futur actualisé qu'elles génèreront, d'autant plus élevé que le climat y est favorable à l'agriculture. Les auteurs justifient cette approche par le fait qu'elle prendrait mieux en compte l'adaptation au climat - en particulier l'optimisation du choix des cultures pour le climat local - que les approches agronomiques. Par exemple, une approche basée sur une modélisation agronomique d'une culture comme le blé risque de surestimer l'impact d'un changement de climat en négligeant le fait que dans un climat différent, il peut être préférable 
de remplacer le blé par une autre culture ou par de l'élevage ${ }^{1}$. Mendelsohn et Massetti [2017] fournissent une synthèse récente des très nombreuses études inspirées par MNS.

Parmi les nombreuses critiques adressées à l'approche ricardienne, l'une des plus courantes concerne la possibilité d'un «biais de prix ». En effet, cette approche n'évalue pas l'effet du climat sur le bien-être des consommateurs de produits agricoles, mais seulement sur celui des producteurs ; or, si le changement climatique réduit l'offre agricole, il en découlera une hausse des prix et une baisse des quantités consommées, impactant les consommateurs. Or, cette hausse de prix augmente la valeur de la production agricole, réduisant voire annulant l'effet négatif de ce changement climatique sur le revenu agricole. Si ce changement climatique est permanent, ce biais s'applique également à l'effet du climat sur la valeur des terres.

Cette critique n'est pas nouvelle puisqu'elle a été mentionnée au moins par Cline [1996], Deressa et al. [2005], Kumar et Parikh [2001], Kurukulasuriya et Ajwad [2007] et Auffhammer et Schlenker [2014]. Dans leur réponse à Cline [1996], Mendelsohn et Nordhaus [1996] admettent que cette critique est théoriquement valide mais ils arguent que ce biais est sans doute quantitativement faible - un argument développé dans Mendelsohn et al. [1999]. Il est vraisemblable qu'en effet, ce biais soit négligeable dans le cas de régressions en coupe instantanée sur un pays développé comme les Etats-Unis, telles que celles menées par MNS. En effet, le réseau de transport y est d'une qualité suffisante pour que les prix des produits agricoles s'égalisent au sein de ce pays.

Cependant, les applications postérieures de cette méthode ont largement porté sur des pays en développement où le transport est nettement plus lent et coûteux, limitant l'égalisation des prix agricoles. De plus, l'information sur les prix n'y est pas toujours parfaitement diffusée, ce qui contribue à limiter cette égalisation. Ainsi, dans son étude portant sur le lien entre les téléphones mobiles et les marchés agricoles au Niger, Aker [2010] a conclu que l'introduction des téléphones mobiles avait réduit la dispersion des prix agricoles entre 10 et $16 \%$, surtout pour les marchés éloignés et ceux reliés par des routes non bitumées.

\footnotetext{
${ }^{1}$ Précisons que certains travaux relevant de l'approche agronomique incluent des options d'adaptation, dont le changement de culture, de variété ou d'itinéraire technique. La critique avancée par MNS conserve néanmoins sa pertinence en ce que dans les approches agronomiques, seules les options d'adaptation explicitement modélisées sont prises en compte.
} 
Plus récemment, Ndiaye, Maître d'Hôtel et Le Cotty [2015], dans leur étude consacrée au rôle de l'éloignement des marchés sur la volatilité des prix du maïs, ont montré qu'il existe un effet robuste et positif des coûts de transport sur la volatilité de ces prix au Burkina Faso.

Par ailleurs, même dans les pays dotés d'un réseau de transport performant, il est possible que le biais de prix soit important pour un autre type d'approche économétrique : celle basée sur une approche en panel, qui s'est développée à la suite de l'article fondateur de Deschênes et Greenstone [2007]. Ces auteurs étudient l'impact sur le revenu agricole des variations interannuelles des températures et des précipitations, pour un panel constitué par les comtés des Etats-Unis. Comme celle de MNS, cette approche a été reprise par de multiples auteurs $^{2}$. Une partie de ces approches utilisent (entre autres) la variabilité interannuelle du revenu agricole pour identifier l'effet des variations interannuelles des variables météorologiques $^{3}$. Or, à moins de travailler à l'échelle d'un pays ou d'une région suffisamment petite et ouverte au commerce international pour être preneuse de prix, les variations de production agricole (y compris celles induites par le climat) vont influencer les prix. Le problème se pose donc pour des études en panel portant sur des producteurs agricoles susceptibles d'influencer les prix mondiaux comme les Etats-Unis (Deschênes et Greenstone, [2007]) ou l'Europe (Vaitkevičiūtė et al., [2019]), pour des cultures comme le maïs ou le blé.

Il y a donc des raisons de penser que le biais de prix peut être significatif dans de nombreux cas d'étude. L'objectif de cet article est de quantifier ce biais de prix dans le cas d'un pays en développement, en l'occurrence le Burkina Faso. Dans une première section, nous clarifions cette notion dans un modèle simple d'équilibre partiel. La deuxième section présente les méthodes économétriques et les données utilisées, la troisième les résultats économétriques et la quatrième les simulations menées pour calculer l'ampleur du biais de prix.

\footnotetext{
${ }^{2}$ Une recherche sur le Web of science menée le 20 décembre 2018 sur les articles de la catégorie « business \& economics » avec les mots clés ("climate change" OR "climatic change" OR "global warming") indique que l'article de MNS est le $3^{\mathrm{e}}$ par le nombre de citations (664 citations) et celui de Deschênes et Greenstone le $26^{\mathrm{e}}$ (303 citations).

${ }^{3} \mathrm{~L}$ 'argument ne s'applique pas à celles de ces approches qui utilisent des effets fixes annuels.
} 


\section{LE BIAIS DE PRIX EN EQUILIBRE PARTIEL : APPROCHE THEORIQUE}

Supposons une variation de la production agricole causée, par exemple, par une évolution des précipitations pendant la saison de culture, dans le cadre d'un modèle d'équilibre partiel linéaire, avec concurrence pure et parfaite. Le raisonnement est ici le même qu'il s'agisse d'un évènement météorologique ponctuel (sur une année) ou d'un changement du climat. Il est le même dans le cas d'une hausse ou d'une baisse de la production.

La Figure 1 représente l'évolution du prix et de la quantité vendue sur un marché en concurrence parfaite, où la production passe de $q_{0}$ à $q_{1}(-50 \%)$ et le prix de $p_{0}$ à $p_{1}(+50 \%)$.

Figure 1. Représentation du biais de prix en équilibre partiel
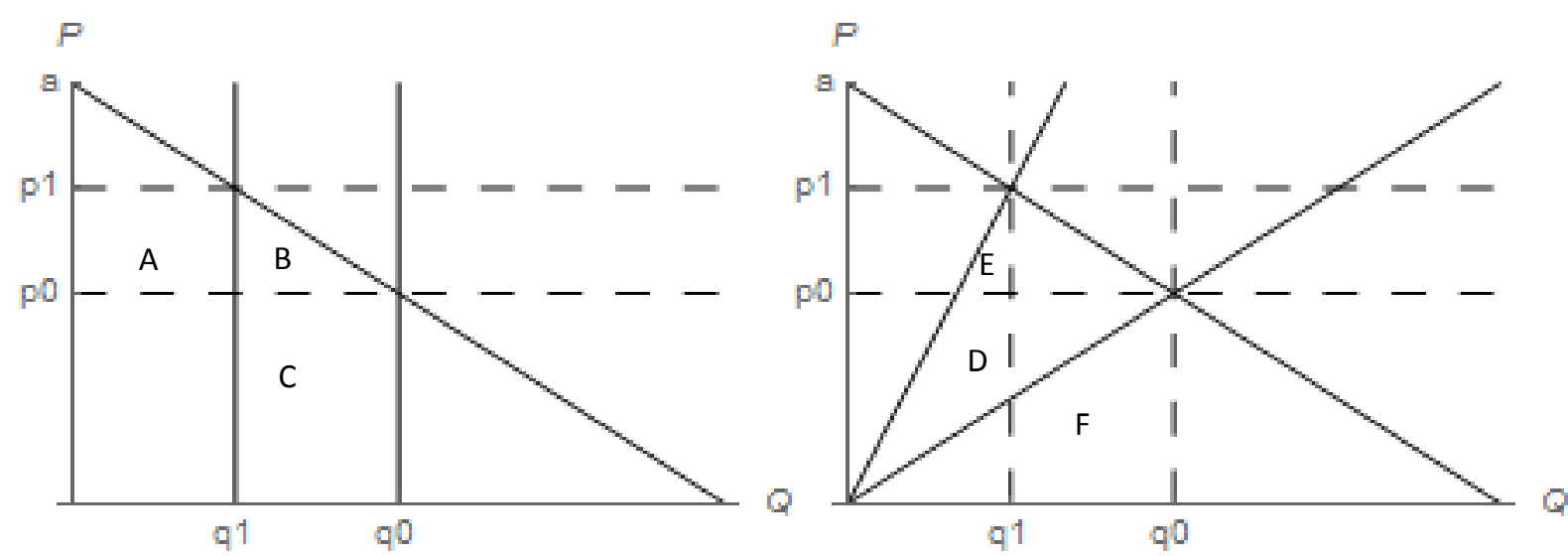

Sur le schéma de gauche, le coût de production total est exogène. La variation du revenu agricole est donc égale à la variation de la valeur de la production, soit la différence entre les deux rectangles $(A-C)^{4}$ :

$$
\Delta v p=p_{1} * q_{1}-p_{0} * q_{0}
$$

La variation de bien-être (qui dans ce cas se limite à la variation de l'utilité du consommateur, puisque le coût de production est exogène) est égale à $(-B-C)$.

\footnotetext{
${ }^{4}$ L'annexe 4 présente une décomposition de ces calculs.
} 


$$
\Delta u c=\frac{\left(q_{1}-q_{0}\right)\left(p_{0}+p_{1}\right)}{2}
$$

En l'absence de variation du prix $\left(p_{0}=p_{1}\right)$ on a bien $\Delta v p=\Delta u c$ : la variation de la valeur de la production mesure correctement la variation de bien-être. C'est le cas si la zone touchée par la variation de la production est « preneuse de prix ».

On peut définir le biais de prix comme la sous-estimation de la variation de bien-être (en valeur absolue) lorsque l'on mesure cette dernière par la variation de la valeur de la production (également en valeur absolue) :

$$
\mathrm{BP}:=|\Delta u c-\Delta v p|=\frac{\left(q_{1}+q_{0}\right)\left|p_{0}-p_{1}\right|}{2}
$$

Graphiquement, ce biais de prix est égal à la surface $(A+\mathrm{B})$.

Sur le schéma de droite, le coût de production marginal crô̂t linéairement avec la production. Sa variation est égale à :

$$
\Delta c p=\frac{p_{1} * q_{1}-p_{0} * q_{0}}{2}
$$

La variation du revenu agricole (autrement dit la marge brute) est égale à la variation de la valeur de la production, comme sur le graphique de gauche, moins la variation du coût de production, soit $(D+E-F)$ :

$$
\Delta r=\Delta v p-\Delta c p=\frac{p_{1} * q_{1}-p_{0} * q_{0}}{2}
$$

La variation du bien-être est quant à elle égale à la variation de l'utilité du consommateur moins la variation du coût de production :

$$
\Delta b e=\Delta u c-\Delta c p=\frac{p_{0} * q_{1}-p_{1} * q_{0}}{2}
$$

Le biais de prix est le même que dans le cas précédent :

$$
\Delta b e-\Delta r=\Delta u c-\Delta v p=B P
$$

Le schéma de droite est celui proposé par Mendelsohn et al. [1999]. Il suppose que le coût de production dépend du volume vendu, donc que les facteurs de production ne sont pas perdus si la production est réduite. On peut donc considérer qu'il représente mieux un impact de long terme, comme une modification du climat, si les facteurs de production peuvent être réalloués suite à cette modification. Dans le présent article, nous estimons l'impact de chocs météorologiques, sur des données annuelles. Qui plus est, nous étudions un pays en 
développement où les cultivateurs ont très peu de coûts de production, hormis le travail familial, non salarié, qui va difficilement trouver une autre occupation suite à un choc météorologique survenant en cours d'année. Dans ce contexte, il se peut que le schéma de gauche soit davantage représentatif que le schéma de droite. En tout état de cause, le biais de prix (en valeur absolue) est le même dans les deux cas, même si ce biais exprimé en proportion de la variation de bien-être (ou en proportion de la variation du revenu agricole), diffère entre ces deux cas.

\section{METHODES ET DONNEES}

\subsection{Description de la zone d'étude}

Le Burkina Faso est un pays en développement parmi les plus pauvres au monde avec un Produit intérieur brut par tête de 792 dollars (en 2014). De plus, environ 80\% de la population vit directement ou indirectement de l'agriculture de subsistance (PNUD [2015]). En outre, de nombreux articles dont ceux de Bassolet et Lutz [1999] et Wourtese et Taylor [2008] confirment que le marché agricole du Burkina Faso est imparfait, c'est-à-dire qu'il souffre de problèmes d'asymétrie d'information entre acheteurs et agriculteurs.

Le Burkina Faso dispose d'un climat de type soudano-sahélien caractérisé par l'alternance d'une saison sèche et d'une saison des pluies. Cette dernière s'étend entre mai et octobre au Sud du pays, et est plus courte au Nord. Le rythme des saisons est déterminé par le déplacement de la zone de convergence intertropicale (SP/ CONAGESE5 ${ }^{5}$, [2001]). Aussi, nous ne prenons pas en compte dans nos régressions les (rares) pluies pendant la saison sèche, entre novembre et avril.

La Figure 2 ci-dessous présente la pluviométrie et la température moyennes sur la période de culture, pour chacune des 45 provinces de notre panel, ainsi que le rendement et le prix moyen. Le gradient Nord-Sud est clairement visible, pour la pluviométrie comme pour les températures et pour le rendement.

\footnotetext{
${ }^{5}$ Secrétariat Permanent du Conseil National pour la Gestion de l'Environnement (Burkina Faso).
} 
Figure 2. Moyennes annuelles des principales variables par province du Burkina

\section{Faso}
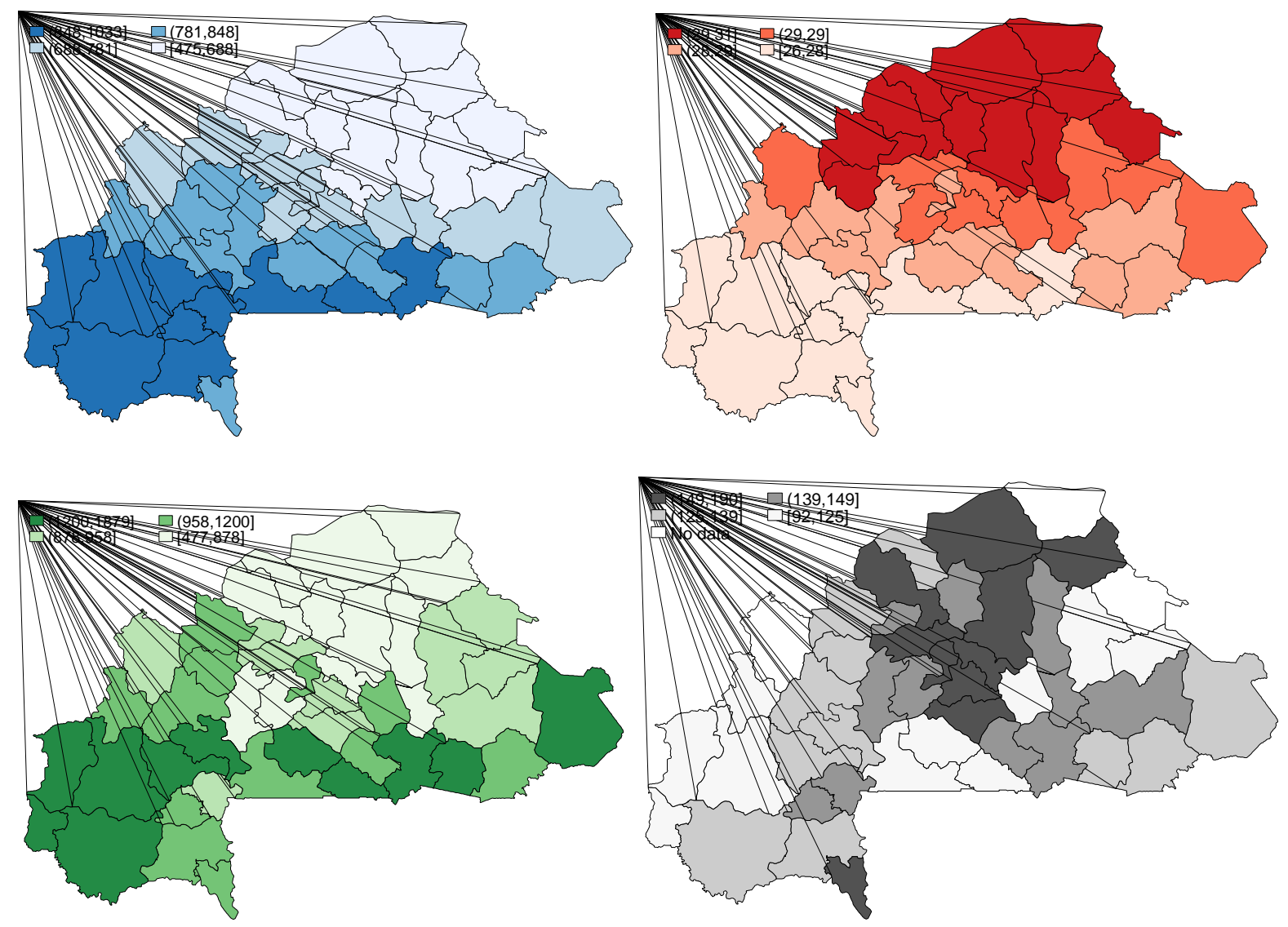

Pluviométrie sur la période de culture (mai à octobre) (en mm, en haut à gauche), température moyenne sur la période de culture (en ${ }^{\circ} \mathrm{C}$, en haut à droite), rendement des céréales (en $\mathrm{kg}$ de grain par hectare, en bas à gauche), prix moyen des céréales (en francs CFA par kg, en bas à droite), en moyenne sur la période 2001-2014 pour chacune des 45 provinces du Burkina Faso

Les céréales retenues (maïs, mil et sorgho) constituent les principales productions végétales au Burkina Faso. Selon le dernier Recensement Général de l’Agriculture (RGA 2008), elles étaient pratiquées sur 4,2 millions d'hectares en 2008, soit environ 73,5\% du total des surfaces cultivées. Le maïs, le mil et le sorgho représentaient alors respectivement $11 \%$, $29 \%$ et $35 \%$ des superficies cultivées.

Au Burkina Faso, le principal intrant de la production des céréales est le travail familial non salarié, les autres facteurs de production étant très limités. Une enquête menée en 2002/2003 a conclu que plus de $80 \%$ des agriculteurs n'utilisaient pas d'engrais (Ouédraogo et al. [2006], Table 1). De plus il est vraisemblable qu'une grande partie des autres aient reçu leurs engrais par les sociétés cotonnières, même s'ils ont pu en utiliser une partie pour leurs 
céréales plutôt que pour la culture du coton, ce qui est courant en Afrique subsaharienne (Leblois et al. [2014]).

\subsection{Méthodes économétriques}

La méthode que nous suivons pour quantifier l'éventuel biais de prix est la suivante. Nous réalisons deux séries de régressions, sur un panel de provinces du Burkina Faso :

(1) Le rendement (c'est-à-dire la production par hectare) sur les variables climatiques ${ }^{6}$.

(2) Le prix des céréales sur le rendement.

La première série de régressions vise à vérifier que les chocs météorologiques impactent le rendement agricole et à quantifier cet impact. On pourrait considérer qu'elle relève davantage de l'agro-météorologie que de l'économie, mais en économie également, ce type de régressions a une longue histoire. Ainsi, Wright [1928], dans ses travaux précurseurs de la méthode des variables instrumentales, utilisait le rendement du coton comme «supply curve shifter (Angrist et Krueger [2001]). La seconde série de régressions vise à étudier si le prix des céréales est influencé par le rendement. Si ces deux séries de régressions sont significatives, nous pourrons conclure à l'existence d'un biais de prix.

Nous utilisons un panel des 45 provinces du Burkina Faso sur 14 années (2001 à 2014). Ce recours à un panel, plutôt qu'à des régressions en coupe instantanée, est justifié par trois raisons. Premièrement, le nombre de provinces est trop faible pour des régressions en coupe instantanée.

Deuxièmement, comme le souligne Vaitkevičiūtė [2018], les régressions en coupe instantanée à la MNS visent à identifier un effet de long terme, et n'ont de sens que si la variable expliquée est la valeur de la terre (qui dépend de la somme actualisée des revenus qu'elle va générer), et non la valeur annuelle de la production ou le revenu agricole annuel. Or, au Burkina Faso comme dans beaucoup d'autres pays pauvres, il n'existe pas de marché des terres généralisé et donc pas de données systématiques sur la valeur des terres.

\footnotetext{
${ }^{6}$ Comme expliqué plus loin (section 2.3), nous retenons parmi les variables explicatives, non le climat à proprement parler mais la réalisation annuelle de ce climat, car nous utilisons des données en panel. Nous retenons le terme «variables climatiques » plutôt que «variables météorologiques » car il est davantage utilisé dans la littérature à laquelle nous faisons référence.
} 
Troisièmement, depuis Deschênes et Greenstone [2007], le recours à des modèles de panel, retenant comme variable dépendante la valeur annuelle de la production ou le revenu agricole annuel par hectare, est de plus en plus fréquent au sein des approches économétriques (Blanc et Schlenker [2017]).

Nous présentons dans le corps de l'article les résultats du modèle à effet aléatoire et ceux du modèle à effet fixe, résultats qui s'avèrent proche. Les différentes équations à estimer séparément (liées aux hypothèses (1) et (2) énoncées plus haut) se définissent comme suit :

$$
\begin{aligned}
& R_{i t}=\alpha * C_{i t}+\beta * C_{i t}^{2}+\gamma_{i}+\mu_{i t} \\
& P_{i t}=\delta * R_{i t-1}+\theta * W_{t}+\eta * I_{t}+\epsilon_{i t}
\end{aligned}
$$

Où $i$ représente la province, $t$ l'année, $R_{i t}$ le rendement, $C_{i t}$ le vecteur des variables climatiques, détaillées plus loin, $P_{i t}$ le prix des céréales, $W_{t}$ le prix mondial des céréales, et $I_{t}$ l'indice des prix à la consommation, ces deux dernières étant des variables de contrôle ${ }^{7}$. $\mu_{i t}$ et $\epsilon_{i t}$ représentent les termes d'erreur des différentes équations ; $\gamma_{i}$ les effets fixes par province présents seulement dans les régressions avec effets fixes. La présence du terme quadratique reflète la relation non-linéaire entre les variables climatiques et les productions agricoles ; on s'attend à ce que le terme linéaire soit positif et le terme quadratique négatif, d'où une relation concave déterminant un optimum climatique.

Dans la seconde équation, le rendement influence les prix avec un décalage d'une année. Cela se justifie parce que les récoltes se déroulent de mi-septembre à décembre, les ventes de céréales s'étalant pendant toute l'année suivante ${ }^{8}$. Le rendement de l'année $t$ influence donc le prix de l'année $t+1$.

\subsection{Données agricoles}

Les quantités de grain récoltées et les superficies cultivées en maïs, mil et sorgho proviennent de la base Agristat (Berg et al. [2009]. Le rendement est par définition le ratio entre quantité de grain récoltée et superficie cultivée. Le rendement total des trois céréales est

\footnotetext{
${ }^{7}$ Dans une partie des régressions, nous remplaçons cette variable par une tendance temporelle linéaire (cf. section 3).

${ }^{8}$ Tristan Le Cotty, communication personnelle.
} 
la somme en $\mathrm{kg}$ des quantités de maïs, mil et sorgho, divisé par la somme des superficies cultivées $^{9}$. Nous présentons également les résultats séparément pour chacune des trois céréales (Annexe 2).

Les prix des céréales au Burkina-Faso ont été fournis par la SO.NA.GE.S.S (Société Nationale de Gestion du Stock de Sécurité alimentaire) à travers l'annuaire des Prix Agricoles et les annuaires statistiques du Burkina Faso.

Le prix mondial du maïs provient de la réserve fédérale de St-Louis, aux Etats-Unis ${ }^{10}$. A notre connaissance, il n'existe pas de prix mondial pour le mil et le sorgho, ces céréales ne faisant l'objet que d'un faible commerce international. L'indice des prix à la consommation au Burkina-Faso provient de l'Université de Sherbooke, au Canada ${ }^{11}$.

Le tableau 1 fournit des statistiques descriptives de ces variables. L'année est codée en prenant 0 pour l'an 2000 .

\subsection{Données climatiques}

Les variables climatiques sont tirées de la base CRU TS 3.23 (Harris et al. [2014]). Ce sont des données en point de grille au pas de temps mensuel et à la résolution spatiale de $0,5^{\circ} \times 0,5^{\circ}$ (soit environ $50 \mathrm{~km}$ par $50 \mathrm{~km}$ à cette latitude), dérivées d'observations par des pluviomètres et des stations météorologiques au sol. Pour attribuer un point de grille à chaque province, nous utilisons la localisation du chef-lieu de la province, qui représente de ce fait le climat de toute la zone. Les disparités climatiques au sein des provinces sont limitées par la superficie réduite de ces dernières (en moyenne $6000 \mathrm{~km}^{2}$ soit à peu de choses près la superficie moyenne d'un département français).

\footnotetext{
${ }^{9}$ Nous avons également testé une agrégation des trois céréales selon leurs valeurs énergétiques selon les taux de conversion suivants : $1 \mathrm{~kg}$ de maïs $=3560$ kilocalories $(\mathrm{kcal}), 1 \mathrm{~kg}$ de mil $=3400 \mathrm{kcal}, 1 \mathrm{~kg}$ de sorgho $=3430$ kcal. Cette conversion nous permet d'obtenir une valeur homogène de l'ensemble des productions (du point de vue de la satisfaction des besoins énergétiques du corps humain). Les résultats étant identiques, nous ne les reproduisons pas ici.

${ }^{10}$ https://fred.stlouisfed.org 11

http://perspective.usherbrooke.ca/bilan/servlet/BMTendanceStatPays?codeTheme=2\&codeStat=FP.CPI.TOTL\& codePays $=$ BFA \&optionsPeriodes $=$ Aucune $\&$ codeTheme $2=2 \& \operatorname{codeStat} 2=x \& \operatorname{codePays} 2=\mathrm{BFA} \& \operatorname{coptionsDetPeriod}$ es=avecNomP\&langue $=\mathrm{fr}$
} 
La plupart des études ricardiennes utilisent communément la température et les précipitations. Cela pose problème dans le contexte du Burkina-Faso car il existe une corrélation négative très nette entre température et précipitations, aussi bien dans l'espace (Figure 2) que dans le temps. La présence de nuages est naturellement nécessaire à la pluie, mais elle génère aussi de l'ombre, ce qui réduit la température en journée. Selon les mois, la corrélation entre températures et pluie va de $-0,3$ à $-0,8$. La corrélation entre les transformations within (l'écart à la moyenne de la province) de ces variables est également forte : entre $-0,3$ et $-0,6$ selon les mois. La forte colinéarité entre ces variables explicatives risque de rendre difficile l'identification du rôle respectif de la pluie et de la température dans la détermination du rendement (Quiggin et Horowitz [1999] ; Martin et Vaitkevičiūtė [2016]).

La figure 3 montre qu'il y a peu de variabilité interannuelle des températures dans chaque province (par rapport aux écarts entre moyennes interannuelles des provinces) mais beaucoup plus pour les pluies. Aussi nous choisissons d'effectuer nos régressions uniquement sur les précipitations. La variable climatique choisie est donc le cumul mensuel des pluies pendant la saison des pluies. La corrélation entre les transformations within des pluies des différents mois de la saison est positive mais faible : entre 0.02 et 0.2 selon les couples de mois. L'utilisation des cumuls de précipitations pour plusieurs mois de l'année n'entrainera donc pas de problème de colinéarité, ce qui est confirmé par le calcul des Variance Inflation Factors (VIF), toujours inférieurs à 1,4, donc très en-dessous du seuil de 10 retenu par Martin et Vaitkevičiūtè [2016] ${ }^{12}$.

En annexe 3, nous présentons les résultats calculés en prenant en compte à la fois les précipitations et les températures; il s'avère que les résultats sur le biais de prix sont similaires, mais les coefficients de la température sont rarement significatifs, et sont moins en phase avec les connaissances agronomiques que ceux de la pluie, qui génèrent les relations concaves attendues.

\footnotetext{
${ }^{12}$ On régresse chacune des variables explicatives sur les autres variables, puis on calcule $\left(1 /\left(1-R^{2}\right)\right)$ ce qui fournit la statistique VIF pour chacune des variables (Martin et Vaitkevičiūtė [2016], p. 26). Plus elle est élevée, plus le problème de colinéarité risque de rendre les résultats erronés.
} 
Figure 3. Variabilité interannuelle de la température et de la pluie, par province
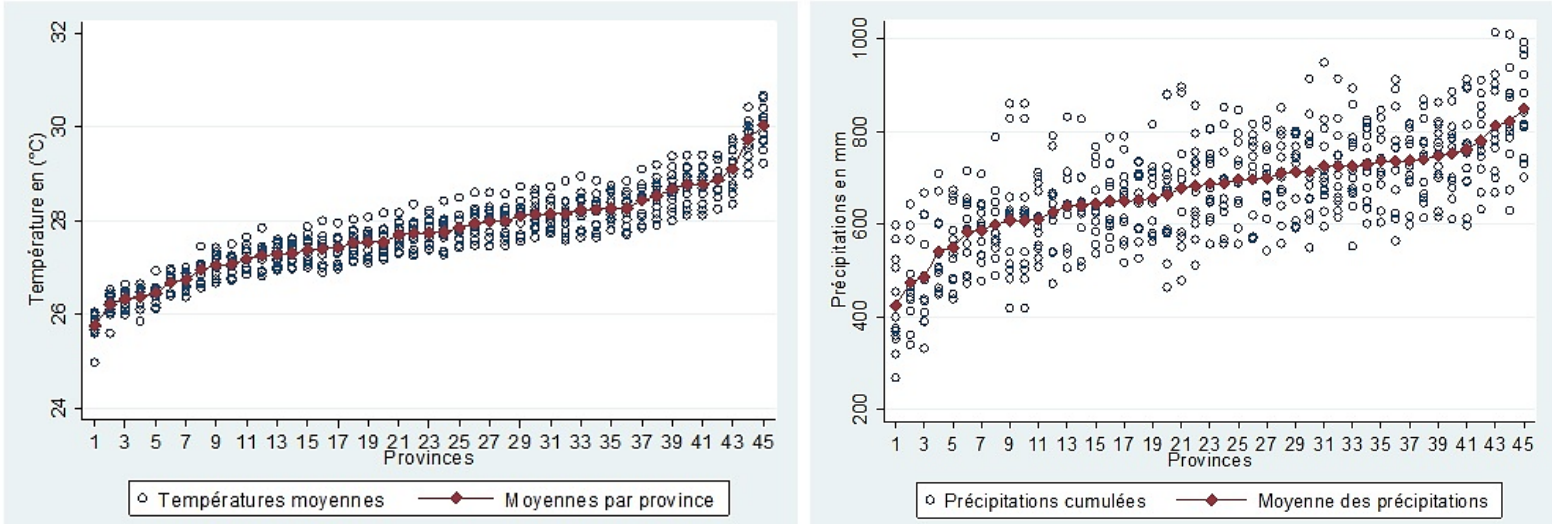

Répartition de la température moyenne (gauche) et des précipitations cumulées (droite) dans les 45 provinces pendant la saison des pluies (juin à septembre) sur 2001-2012. En abscisse, chaque chiffre correspond à une province. Les correspondances (différentes pour température et précipitation) sont indiquées en annexe 1.

Tableau 1. Valeurs moyennes des principales variables

\begin{tabular}{|l|r|r|r|r|r|r|c|}
\hline & mai & juin & juillet & août & sept. & octobre & source \\
\hline pluies $(\mathrm{mm})$ & 57 & 106 & 182 & 231 & 151 & 45 & CRU TS \\
\hline températures $\left({ }^{\circ} \mathrm{C}\right)$ & 32 & 30 & 27 & 27 & 27 & 29 & CRU TS \\
\hline & maïs & mil & sorgho & moyenne & & & \\
\hline rendement (kg/ha) & 1321 & 892 & 1030 & 1042 & & & Agristat \\
\hline prix (FCFA/kg) & 133 & 158 & 138 & 140 & & & SO.NA.GE.S.S \\
\hline $\begin{array}{l}\text { prix mondial du } \\
\text { maïs }\end{array}$ & 147 & & & & & & Fred St.Louis \\
\hline
\end{tabular}

\subsection{Méthode d'estimation}

Avant de présenter les résultats, il convient de procéder aux différents tests d'hypothèses. Les tests de racine unitaire de Dickey et Fuller (ADF) (Dickey et Fuller [1979]) concluent que les variables explicatives (les précipitations et les températures) sont stationnaires en niveau. Les rendements sont aussi stationnaires. Le prix des céréales est stationnaire en différence première. Ainsi, nous pouvons pour l'équation (1), continuer la procédure d'estimation. Pour l'équation (2), nous n'avons pas besoin de passer à des tests de cointégration puisque les variables explicatives, c'est-à-dire les pluies, sont stationnaires en 
niveau et que le prix l'est en différence première. Nous effectuons donc l'estimation en tenant compte de la stationnarité en différence première de l'indice de prix.

Les différentes équations présentent des problèmes d'hétéroscédasticité. Nous les corrigeons en utilisant l'estimateur de White [1980] pour la matrice de variance et covariance des estimateurs. Le logiciel STATA 13 est utilisé pour les régressions.

Nous présentons les résultats des modèles à effets aléatoires et à effets fixes par province, avec à chaque fois une variante sans tendance temporelle et une autre avec tendance temporelle linéaire, de manière à prendre en compte le progrès technique, qui peut se matérialiser en particulier à travers l'amélioration des variétés cultivées.

\section{RESULTATS}

Concernant l'estimation du rendement, les quatre modèles économétriques donnent des résultats très proches. Dans chacun d'entre eux, les coefficients des pluies en juillet et en octobre ne sont pas statistiquement significatifs au seuil de 5\%. Pour juillet, l'explication est sans doute que les plantes ne souffrent pas de pénurie d'eau en ce mois situé au cœur de la saison des pluies, et qui ne correspond pas aux phases de croissance des céréales les plus sensibles à la sécheresse. Quant à octobre, des pluies abondantes peuvent y avoir un effet aussi bien positif (allonger la période de croissance des plantes) que négatif (favoriser des moisissures $)^{13}$. Aussi, nous présentons dans le Tableau 2 les résultats sans les mois de juillet et d'octobre.

\footnotetext{
${ }^{13}$ Nous remercions Tristan Le Cotty, chercheur CIRAD à l'époque en poste au Burkina Faso, pour ses éclairages sur ces points.
} 
Tableau 2. Résultats des estimations du rendement pour l'ensemble des trois céréales

\begin{tabular}{|c|c|c|c|c|c|c|c|c|}
\hline $\begin{array}{l}\text { Modèle } \rightarrow \\
\text { Variable } \downarrow\end{array}$ & RE & & RE_TT & & FE & & FE_TT & \\
\hline Pluie mai & 1.141 & & 1.779 & $*$ & 0.527 & & 1.120 & \\
\hline Pluie mai ${ }^{2}$ & 0.002 & & -0.002 & & 0.003 & & -0.001 & \\
\hline Pluie juin & 4.671 & $* * *$ & 4.074 & $* * *$ & 3.857 & $* *$ & 3.128 & $*$ \\
\hline Pluie juin ${ }^{2}$ & -0.018 & $* * *$ & -0.014 & $* *$ & -0.015 & $*$ & -0.011 & $*$ \\
\hline Pluie août & 2.037 & $* *$ & 1.693 & $*$ & 1.914 & $* *$ & 1.522 & $*$ \\
\hline Pluie août ${ }^{2}$ & -0.004 & $* *$ & -0.004 & $* *$ & -0.004 & $* *$ & -0.004 & $*$ \\
\hline Pluie septembre & 4.271 & $* * *$ & 4.106 & $* * *$ & 4.212 & $* * *$ & 3.998 & $* * *$ \\
\hline $\begin{array}{l}\text { Pluie } \\
\text { septembre } 2\end{array}$ & -0.010 & $* * *$ & -0.010 & $* * *$ & -0.010 & $* * *$ & -0.010 & $* * *$ \\
\hline Année & & & 8.809 & $* *$ & & & 9.105 & $* *$ \\
\hline Constante & 82.831 & & 94.397 & & 204.959 & & 234.127 & \\
\hline $\begin{array}{l}\text { Effets fixes par } \\
\text { province }\end{array}$ & Non & & Non & & Oui & & Oui & \\
\hline $\mathrm{N}$ & 551 & & 551 & & 551 & & 551 & \\
\hline $\mathrm{R}^{2}$ within & 0.098 & & 0.125 & & 0.102 & & 0.131 & \\
\hline $\mathrm{R}^{2}$ between & 0.485 & & 0.527 & & 0.415 & & 0.456 & \\
\hline $\mathrm{R}^{2}$ overall & 0.273 & & 0.283 & & 0.233 & & 0.234 & \\
\hline
\end{tabular}

$* \mathrm{p}<.05 ; * * \mathrm{p}<.01 ; * * * \mathrm{p}<.001$

RE : modèle à effets aléatoires. RE_TT : avec tendance temporelle.

FE : modèle à effets fixes par province. FE_TT : avec tendance temporelle

Les relations sont toujours concaves, comme attendu, et nous avons vérifié que pour chaque mois, la pluie moyenne se situe toujours dans la partie croissante de la courbe. Les coefficients sont particulièrement élevés pour juin et septembre, soit en début et en fin de la saison des pluies : le rendement augmente d'environ $4 \mathrm{~kg} / \mathrm{ha}$ pour chaque $\mathrm{mm}$ de pluie en plus par mois. De faibles pluies en juin ou en septembre signifient en effet une saison des pluies courte, d'où de mauvaises récoltes. On note également que la tendance temporelle est positive, comme attendu, et significative, traduisant une hausse du rendement de $9 \mathrm{~kg} / \mathrm{ha} / \mathrm{an}$, 
toutes choses égales par ailleurs. Le coefficient de mai n'est significatif au seuil de 5\% que pour le modèle à effets aléatoires avec tendance temporelle. L'explication de cette plus faible significativité statistique est sans doute que la saison de culture démarre en mai au Sud, mais seulement en juin dans le reste du pays, d'où une influence limitée des pluies en mai.

Les valeurs des coefficients des pluies sont légèrement plus faibles dans les modèles avec effets fixes. Une explication vraisemblable est que les effets fixes neutralisent la corrélation systématique pluie-rendement entre les différentes provinces, qu'on constate Figure 2. De ce point de vue, on peut considérer que les modèles à effets fixes mesurent un effet de court terme (celui des fluctuations annuelles de la saison des pluies) tandis que les modèles à effets aléatoires prennent en compte également l'effet des différences de climat entre provinces, qu'on peut interpréter, à la suite de MNS, comme un effet de long terme. Les valeurs des coefficients sont par ailleurs légèrement plus faibles dans les modèles avec tendance temporelle. Les valeurs des différents $\mathrm{R}^{2}$ indiquent que les différents modèles expliquent environ $10 \%$ de la variance temporelle environ la moitié de la variance entre provinces, et un peu plus du quart de la variance totale.

En annexe, nous présentons les résultats des estimations du rendement réalisées séparément pour chacune des trois céréales (Tableaux A1 à A3). Les résultats sont généralement proches de ceux pour l'ensemble des trois céréales. Parmi les différences notables, les coefficients des pluies sont plus élevés pour le maïs, ce qui est cohérent avec le fait que cette céréale est moins robuste à la sécheresse que le mil et le sorgho. Pour le sorgho, les pluies en mai sont plus déterminantes que celles en juin et en août. Enfin, la tendance temporelle n'est significative au seuil de 5\% pour le rendement d'aucune des céréales prises séparément, alors qu'elle l'est pour le rendement agrégé des trois céréales. L'explication provient de la hausse au cours de la période de la part du maïs, dont le rendement en grain est plus élevé que celui du mil et du sorgho (Tableau 1). Autrement dit, la hausse du rendement en grain au cours du temps est influencée par un effet de composition.

Sur la zone soudano-sahélienne qui comprend le Burkina-Faso, les résultats des modèles climatiques synthétisés dans les $4^{\mathrm{e}}$ et $5^{\mathrm{e}}$ rapports de synthèse du GIEC ne convergent pas quant à l'évolution des précipitations au $21^{\mathrm{e}}$ siècle. Sur la période 2031-2050 comme sur la 
période 2071-2090, ces précipitations pourraient varier de -20\% à $+20 \%$ par rapport à 19611990 (Sultan et al. [2013], Figure $2^{14}$ ).

Le tableau 3 présente l'évolution du rendement du fait d'une baisse de la pluie dans la même proportion pour chaque mois, pour les quatre modèles économétriques, sur la base des coefficients indiqués au Tableau 2. Une baisse d'un pour cent de la pluie entraîne une baisse du rendement du même ordre. L'effet est plus élevé pour le maïs et plus faible pour le mil ; il est plus faible pour les modèles à effets fixes et pour ceux qui incluent une tendance temporelle, en cohérence avec les coefficients indiqués au Tableau 2. Pour le mil et le sorgho, ces effets quantitatifs sont cohérents avec ceux obtenus par Sultan et al. ([2013], Figure 2) à partir d'un modèle agronomique mécaniste, soit une baisse du rendement de 10 à $20 \%$ pour une baisse de la pluie de $20 \%$, à température constante.

\footnotetext{
${ }^{14}$ Dans cet article, la zone soudano-sahélienne est définie par les limites suivantes $: 2,5^{\circ}$ à $15^{\circ}$ de latitude nord, $19^{\circ}$ de longitude ouest à $17^{\circ}$ de longitude est.
} 
Tableau 3. Variation du rendement en fonction de la pluie

\begin{tabular}{|c|c|c|c|c|c|}
\hline & 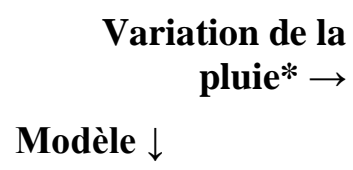 & $-20 \%$ & $-15 \%$ & $-10 \%$ & $-5 \%$ \\
\hline \multirow[t]{4}{*}{3 céréales } & RE & $-20.4 \%$ & $-15.3 \%$ & $-10.2 \%$ & $-5.1 \%$ \\
\hline & RE_TT & $-20.1 \%$ & $-15.1 \%$ & $-10.1 \%$ & $-5.0 \%$ \\
\hline & $\mathbf{F E}$ & $-17.7 \%$ & $-13.3 \%$ & $-8.9 \%$ & $-4.4 \%$ \\
\hline & FE_TT & $-17.1 \%$ & $-12.8 \%$ & $-8.5 \%$ & $-4.3 \%$ \\
\hline \multirow[t]{4}{*}{ Maïs } & $\mathbf{R E}$ & $-32.9 \%$ & $-24.7 \%$ & $-16.5 \%$ & $-8.2 \%$ \\
\hline & RE_TT & $-32.6 \%$ & $-24.4 \%$ & $-16.3 \%$ & $-8.1 \%$ \\
\hline & $\mathbf{F E}$ & $-31.3 \%$ & $-23.5 \%$ & $-15.7 \%$ & $-7.8 \%$ \\
\hline & FE_TT & $-30.9 \%$ & $-23.2 \%$ & $-15.4 \%$ & $-7.7 \%$ \\
\hline \multirow[t]{4}{*}{ Mil } & $\mathbf{R E}$ & $-16.4 \%$ & $-12.3 \%$ & $-8.2 \%$ & $-4.1 \%$ \\
\hline & RE_TT & $-16.6 \%$ & $-12.4 \%$ & $-8.3 \%$ & $-4.1 \%$ \\
\hline & $\mathbf{F E}$ & $-16.0 \%$ & $-12.0 \%$ & $-8.0 \%$ & $-4.0 \%$ \\
\hline & FE_TT & $-16.1 \%$ & $-12.1 \%$ & $-8.1 \%$ & $-4.0 \%$ \\
\hline \multirow[t]{4}{*}{ Sorgho } & $\mathbf{R E}$ & $-19.0 \%$ & $-14.2 \%$ & $-9.5 \%$ & $-4.7 \%$ \\
\hline & RE_TT & $-18.9 \%$ & $-14.2 \%$ & $-9.4 \%$ & $-4.7 \%$ \\
\hline & $\mathbf{F E}$ & $-17.4 \%$ & $-13.0 \%$ & $-8.7 \%$ & $-4.3 \%$ \\
\hline & FE_TT & $-17.3 \%$ & $-13.0 \%$ & $-8.6 \%$ & $-4.3 \%$ \\
\hline
\end{tabular}

* Variation de la pluie par rapport à la moyenne 2001-2014, pour chaque province, d'un même pourcentage pour les mois de mai, juin, août et septembre.

RE : modèle à effets aléatoires. RE_TT : avec tendance temporelle.

FE : modèle à effets fixes par province. FE_TT : avec tendance temporelle.

Le tableau 4 présente le résultat de l'estimation de l'équation (2), expliquant le prix moyen des trois céréales. Parmi les variables de contrôle, nous incluons soit l'indice des prix à la consommation, soit une tendance temporelle linéaire, mais pas ces deux variables simultanément car elles sont trop corrélées. 
Tableau 4. Résultats des estimations du prix pour l'ensemble des trois céréales

\begin{tabular}{|c|c|c|c|c|c|c|c|c|}
\hline $\begin{array}{c}\text { Modèle } \rightarrow \\
\text { Variable } \downarrow\end{array}$ & RE & & RE_TT & & FE & & FE_TT & \\
\hline $\begin{array}{l}\text { Rendement en t- } \\
1\end{array}$ & $\begin{array}{c}-0.033 \\
(-.043 ;- \\
.024)\end{array}$ & $* * *$ & $\begin{array}{c}-0.034 \\
(-.043 ;- \\
.024)\end{array}$ & $* * *$ & $\begin{array}{c}-0.026 \\
(-.042 ;- \\
.010)\end{array}$ & $* *$ & $\begin{array}{c}-0.026 \\
(-.042 ;- \\
.011)\end{array}$ & $* *$ \\
\hline $\begin{array}{l}\text { Prix mondial du } \\
\text { maïs }\end{array}$ & 0.20 & $* * *$ & 0.30 & $* * *$ & 0.21 & $* * *$ & 0.31 & $* * *$ \\
\hline $\begin{array}{l}\text { Indice des prix à } \\
\text { la consommation }\end{array}$ & 1.00 & $* * *$ & & & 0.94 & $* * *$ & & \\
\hline Année & & & 0.96 & $* *$ & & & 0.64 & \\
\hline Constante & 50.27 & $* * *$ & 122.79 & $* * *$ & 48.22 & $* * *$ & 116.24 & $* * *$ \\
\hline $\begin{array}{l}\text { Effets fixes par } \\
\text { province }\end{array}$ & Non & & Non & & Oui & & Oui & \\
\hline $\mathrm{N}$ & 439 & & 439 & & 439 & & 439 & \\
\hline $\mathrm{R}^{2}$ within & 0.37 & & 0.34 & & 0.37 & & 0.34 & \\
\hline $\mathrm{R}^{2}$ between & 0.58 & & 0.56 & & 0.57 & & 0.57 & \\
\hline $\mathrm{R}^{2}$ overall & 0.40 & & 0.38 & & 0.39 & & 0.37 & \\
\hline
\end{tabular}

$* \mathrm{p}<.05 ; * * \mathrm{p}<.01 ; * * * \mathrm{p}<.001$. L'intervalle de confiance à $95 \%$ est indiqué entre parenthèses pour la variable d'intérêt (le rendement en t-1).

RE : modèle à effets aléatoires. RE_TT : avec tendance temporelle.

FE : modèle à effets fixes par province. FE_TT : avec tendance temporelle.

Tous les coefficients sont du signe attendu et significatifs au seuil de $1 \%$, sauf la tendance temporelle en présence d'effets fixes. Chaque modèle explique plus du tiers de la variance temporelle $\left(\mathrm{R}^{2}\right.$ within $\left.>0.33\right)$ et plus de la moitié de la variance inter-provinces $\left(\mathrm{R}^{2}\right.$ between $>0.5$ ). Bien que le prix mondial du maïs ait un effet positif sur le prix intérieur des céréales, l'hypothèse de petit pays ne peut être retenue : l'élasticité du prix domestique au prix mondial n'est que de 0,2 à 0,31 et le rendement de l'année précédente a bien un effet significatif sur le prix domestique.

Les résultats des régressions menées séparément sur les trois céréales (Tableaux A4 à A6) sont proches de ceux des régressions menées sur le prix moyen des céréales. 
Le tableau 5 présente la variation du prix des céréales en fonction de celle de la pluie. Ces résultats sont obtenus en calculant le rendement en fonction de la pluie avec l'équation (1) et les coefficients du Tableau 2, puis le prix en fonction du rendement, avec l'équation (2) et les coefficients du Tableau 4.

Une baisse de la pluie de $20 \%$ entraîne une hausse du prix des céréales d'environ $5 \%$ en utilisant un modèle à effets aléatoires (intervalle de confiance à 95\%:3,5 à 7\%), et environ $4 \%$ (intervalle de confiance : 1,5\% à 7\%) en utilisant un modèle à effets fixes. La présence ou non d'une tendance temporelle dans les régressions ne change que très peu ces résultats. 
Tableau 5. Variation du prix moyen des trois céréales, en fonction de la pluie

\begin{tabular}{|c|c|c|c|c|c|}
\hline & $\begin{array}{r}\text { Variation de la } \\
\text { pluie* }\end{array}$ & $-20 \%$ & $-15 \%$ & $-10 \%$ & $-5 \%$ \\
\hline \multirow{4}{*}{$\begin{array}{l}\text { Meilleure } \\
\text { estimation }\end{array}$} & RE & $5.0 \%$ & $3.8 \%$ & $2.5 \%$ & $1.3 \%$ \\
\hline & RE_TT & $5.0 \%$ & $3.8 \%$ & $2.5 \%$ & $1.3 \%$ \\
\hline & FE & $3.9 \%$ & $3.0 \%$ & $2.0 \%$ & $1.0 \%$ \\
\hline & FE_TT & $3.9 \%$ & $2.9 \%$ & $2.0 \%$ & $1.0 \%$ \\
\hline \multirow{4}{*}{$\begin{array}{l}\text { Borne } \\
\text { supérieure*** }\end{array}$} & $\mathbf{R E}$ & $6.9 \%$ & $5.2 \%$ & $3.5 \%$ & $1.7 \%$ \\
\hline & RE_TT & $6.9 \%$ & $5.2 \%$ & $3.5 \%$ & $1.7 \%$ \\
\hline & FE & $7.1 \%$ & $5.3 \%$ & $3.6 \%$ & $1.8 \%$ \\
\hline & FE_TT & $7.1 \%$ & $5.3 \%$ & $3.6 \%$ & $1.8 \%$ \\
\hline \multirow{4}{*}{$\begin{array}{l}\text { Borne } \\
\text { inférieure }{ }^{* *}\end{array}$} & $\mathbf{R E}$ & $3.4 \%$ & $2.5 \%$ & $1.7 \%$ & $0.8 \%$ \\
\hline & RE_TT & $3.4 \%$ & $2.5 \%$ & $1.7 \%$ & $0.8 \%$ \\
\hline & $\mathbf{F E}$ & $1.3 \%$ & $1.0 \%$ & $0.7 \%$ & $0.3 \%$ \\
\hline & FE_TT & $1.5 \%$ & $1.1 \%$ & $0.7 \%$ & $0.4 \%$ \\
\hline
\end{tabular}

* Variation de la pluie par rapport à la moyenne 2001-2014, pour chaque province, d'un même pourcentage pour les mois de mai, juin, août et septembre.

** Résultats des simulations obtenues en utilisant les bornes inférieures et supérieures de l'intervalle de confiance à $95 \%$ présentées au Tableau 4 .

RE : modèle à effets aléatoires. RE_TT : avec tendance temporelle.

FE : modèle à effets fixes par province. FE_TT : avec tendance temporelle.

\section{QUANTIFICATION DU BIAIS DE PRIX}

Dans cette partie, nous calculons le biais de prix BP présenté dans la première section, à l'aide des estimations économétriques présentées en section 3. Les valeurs de $p_{0}$ et $q_{0}$ sont obtenues par les équations estimées pour les valeurs moyennes de la pluie, les valeurs $p_{1}$ et $q_{1}$ pour les valeurs moyennes, diminuées du pourcentage indiqué dans le Tableau 6.

Ce biais est exprimé en pourcentage du revenu agricole, sous l'hypothèse de linéarité du coût marginal de production, comme dans Mendelsohn et al. [1999]. Sous cette hypothèse, le 
revenu agricole est égal à la moitié de la valeur de la production (section 1); pour obtenir le biais en pourcentage de cette dernière, il suffit donc de diviser par deux les valeurs du Tableau 6.

Quantitativement, le biais est important : une étude économétrique qui négligerait la hausse du prix entraînée par la baisse de la pluie sous-estimerait l'effet réel d'environ $50 \%$ à $70 \%$ (selon le modèle économétrique et l'ampleur de la baisse de la pluie), si l'on prend l'estimation centrale du coefficient du rendement dans l'équation du prix. Le biais est plus faible si l'on se base sur les régressions avec effets fixes par province, et diminue (en pourcentage) avec l'ampleur du choc. L'intervalle de confiance à 95\% est nettement plus étendu avec les régressions à effets fixes.

En tout état de cause, le biais est nettement plus important que mentionné par Mendelsohn et al. [1999], pour qui il ne devrait pas dépasser quelques pourcents. Soulignons que leurs calculs ne se basent pas sur des estimations économétriques et qu'ils concernent les Etats-Unis, pays où le réseau de transport est bien plus développé que le Burkina Faso ; rien ne dit que nos conclusions s'appliquent également à des pays développés, puisque les faibles coûts de transports y homogénéisent les prix.

Le Tableau A8 présente le biais de prix en pourcentage du revenu agricole pour chaque céréale séparément. Pour le maïs, le résultat est supérieur à celui pour l'ensemble des céréales alors que l'inverse est vrai pour le sorgho et surtout pour le mil, conformément aux coefficients qui indiquent la réponse du prix en fonction de la pluie, présentés au Tableau A7. Pour le maïs, avec les modèles à effets aléatoires, le biais de prix est supérieur à 100\%, ce qui signifie que la perte de bien-être est plus du double de la perte de revenu agricole. 
Tableau 6. Biais de prix en pourcentage du revenu agricole

\begin{tabular}{|c|c|c|c|c|c|}
\hline & 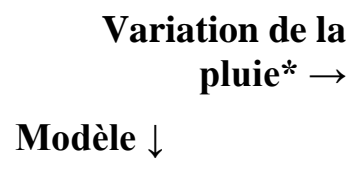 & $-20 \%$ & $-15 \%$ & $-10 \%$ & $-5 \%$ \\
\hline \multirow{4}{*}{$\begin{array}{l}\text { Meilleure } \\
\text { estimation }\end{array}$} & $\mathbf{R E}$ & $63 \%$ & $66 \%$ & $69 \%$ & $72 \%$ \\
\hline & RE_TT & $64 \%$ & $66 \%$ & $69 \%$ & $72 \%$ \\
\hline & $\mathbf{F E}$ & $47 \%$ & $49 \%$ & $50 \%$ & $52 \%$ \\
\hline & FE_TT & $47 \%$ & $48 \%$ & $50 \%$ & $51 \%$ \\
\hline \multirow{4}{*}{$\begin{array}{l}\text { Borne } \\
\text { supérieure*** }\end{array}$} & $\mathbf{R E}$ & $99 \%$ & $104 \%$ & $110 \%$ & $116 \%$ \\
\hline & RE_TT & $99 \%$ & $104 \%$ & $109 \%$ & $115 \%$ \\
\hline & $\mathbf{F E}$ & $104 \%$ & $109 \%$ & $114 \%$ & $120 \%$ \\
\hline & FE_TT & $104 \%$ & $109 \%$ & $114 \%$ & $119 \%$ \\
\hline \multirow{4}{*}{$\begin{array}{l}\text { Borne } \\
\text { inférieure } * *\end{array}$} & $\mathbf{R E}$ & $39 \%$ & $40 \%$ & $41 \%$ & $43 \%$ \\
\hline & RE_TT & $38 \%$ & $40 \%$ & $41 \%$ & $43 \%$ \\
\hline & $\mathbf{F E}$ & $14 \%$ & $14 \%$ & $15 \%$ & $15 \%$ \\
\hline & FE_TT & $15 \%$ & $16 \%$ & $16 \%$ & $17 \%$ \\
\hline
\end{tabular}

* Variation de la pluie par rapport à la moyenne 2001-2014, pour chaque province, d'un même pourcentage pour les mois de mai, juin, août et septembre.

** Résultats des simulations obtenues en utilisant les bornes inférieures et supérieures de l'intervalle de confiance à $95 \%$ du Tableau

RE : modèle à effets aléatoires. RE_TT : avec tendance temporelle.

FE : modèle à effets fixes par province. FE_TT : avec tendance temporelle.

\section{CONCLUSION}

Depuis Cline [1996], plusieurs auteurs ont discuté l'ampleur du biais de prix dont pourraient souffrir les estimations économétriques consacrées à l'impact du climat sur l'agriculture, mais cet effet n'avait jamais été quantifié économétriquement. Nous le quantifions dans le cas de la culture des céréales (maïs, mil et sorgho) au Burkina Faso à l'aide de différents modèles de panel. L'ampleur du biais dépend des hypothèses sur les coûts agricoles, du modèle économétrique retenu et de l'importance des effets climatiques eux- 
mêmes, mais en tout état de cause, il est important : négliger la variation des prix et estimer l'impact du choc climatique en mesurant le revenu agricole amène à sous-estimer l'impact réel d'un choc météorologique ou climatique d'environ 20 à $70 \%$. Ce résultat est robuste à différentes spécifications économétriques et à différents types de chocs climatiques.

Le biais de prix est peut-être négligeable pour des pays développés où les réseaux de transports assurent l'égalisation des prix agricoles, et pour des pays «preneurs de prix » sur les marchés mondiaux, mais on peut s'attendre à ce qu'il soit important pour d'autres pays à revenu faible. Or, ce sont les pays dont la sécurité alimentaire est le plus menacée par le changement climatique.

Une piste de recherche future consisterait à mener ce type d'estimations sur plusieurs pays à la fois, pour quantifier le biais de prix dans un tel contexte. On peut en effet s'attendre à ce que les écarts de prix soient plus élevés si l'on prend en compte plusieurs pays, amplifiant le biais de prix. La disponibilité grandissante de données de prix agricoles à travers les programmes de la $\mathrm{FAO}^{15}$ et de l'US AID $^{16}$ permet d'envisager ce type d'études dans les années qui viennent. D’un autre côté, le Burkina Faso est un pays enclavé ; dans des pays bénéficiant de ports de haute mer, il se peut que l'influence des prix internationaux soit supérieure, d'où un biais de prix plus faible. Là encore, de nouvelles études seraient bienvenues.

15 FAO Global Information and Early Warning System (GIEWS). http://www.fao.org/giews/foodprices/tool/public/\#/dataset/domestic.

\footnotetext{
${ }^{16}$ Famine Early Warning System Network. http://fews.net/fr/fews-data/337.
} 


\section{REFERENCES}

AKER C.J. [2010], «Information from markets near and far: Mobile phones and agricultural markets in Niger ». American Economic Journal: Applied Economics, 2(3), 46-59.

ANGRIST J.D. et KRUEGER A.B. [2001], «Instrumental Variables and the Search for Identification: From Supply and Demand to Natural Experiments ». Journal of Economic Perspectives, 15 (4): 69-85

AufFHAMmer M. et SCHLENKER W. [2014], «Empirical studies on agricultural impacts and adaptation ». Energy Economics, 46, 555-561.

BASSOLET B. et LuTZ C. [1999], «Information service and integration of cereal markets in Burkina Faso ». Journal of African Economies. 8(1), 31-51.

Berg A., Quirion P. et Sultan B. [2009], «Weather-index drought insurance in BurkinaFaso: assessment of its potential interest to farmers ». Weather, Climate and Society, 1(1): 7184 , October.

BLANC E. et REILLY J. [2017], «Approaches to assessing climate change impacts on agriculture: an overview of the debate ». Review of Environmental Economics and Policy, $11(2), 247-257$.

BlAnC E. et SCHLENKER W. [2017], «The use of panel models in assessments of climate impacts on agriculture ». Review of Environmental Economics and Policy, 11(2), 258-279.

Cline W. R. [1996], «The impact of global warming on agriculture: Comment », The American Economic Review 86(5), 1309-1301.

Deressa T., Hassan, R. et PoOnYth, D. [2005], « Measuring the impact of climate change on South African agriculture: the case of sugar-cane growing regions ». Agrekon 44(4):524542

Deschenes O. et Greenstone M. [2007], «The Economic Impacts of Climate Change: Evidence from Agricultural Profits and Random Fluctuations in Weather ». American Economic Review, 97 (1), 354-385.

DiCKEY D.A. et FuLLER W.A. [1979], « Distribution of the estimators for autoregressive time series with a unit root ». Journal of the American statistical association, 74 (366a), 427-431. 
HARris I., Jones P.D., OSBORN T.J. et LISTER D.H. [2014], « Updated high-resolution grids of monthly climatic observations - the CRU TS3.10 Dataset ». International Journal of Climatology 34, 623-642

KUMAR K. K. et PARIKH J. [2001], «Indian agriculture and climate sensitivity ». Global Environmental Change, 11(2), 147-154.

Kurukulasuriya P. et AJWAD M.I. [2007], «Application of the Ricardian technique to estimate the impact of climate change on smallholder farming in Sri Lanka ». Climate Change 81(1):39-59

Leblois, A. Quirion P. et Sultan B. [2014], « Price vs. weather shock hedging for cash crops: ex ante evaluation for cotton producers in Cameroon », Ecological Economics 101:6780

MARTin E. et VAitKeviciute J. [2016], «Mesure de l'impact du changement climatique sur l'agriculture de Côte-d'Or ». Économie Rurale 355: 21-48

Mendelsohn R. O. et MassetTi E. [2017], «The use of cross-sectional analysis to measure climate impacts on agriculture: theory and evidence ». Review of Environmental Economics and Policy, 11(2), 280-298.

MENDELSOHN R. et NoRdHAus W. [1996], « The Impact of Global Warming on Agriculture: A Ricardian Analysis: Reply ». The American Economic Review 86(5): 1012-15.

Mendelsohn R., Nordhaus W. et Shaw D. [1994], «The impact of global warming on agriculture: A Ricardian analysis ». American Economic Review 84, 753-771.

MENDELSOHN R., NoRdHAUS W. et SHAW D. [1999], The impact of climate variation on US agriculture (pp. 55-74). Cambridge University Press, Cambridge, United Kingdom and New York, NY.

Ndiaye M., Maître D'Hôtel E. et Le CotTy T. [2015], « Maize price volatility: does market remoteness matter? » Policy Research Working Paper 7202World Bank Group

Oú́draogo M., Some L. et Dembele Y. [2006], «Economic impact assessment of climate change on agriculture in Burkina Faso: A Ricardian Approach ». CEEPA DP24, University of Pretoria, South Africa.

PNUD (Programme des Nations Unies pour le Développement) [2015], Rapport Annuel 2014 du PNUD Burkina Faso. 
QuigGin J., HoRowitz J. K. [1999], «The Impact of Global Warming on Agriculture: A Ricardian Analysis: Comment ». American Economic Review, 89 (4), pp. 1044-45.

Roudier P., Sultan B., Quirion P. et BERG A. [2011], «The impact of future climate change on West African agriculture: a review », Global Environmental Change, 21(3): 10731083 doi:10.1016/j.gloenvcha.2011.04.007

Rosenzweig C., Parry M., Frohberg K. et Fisher G. [1993], Climate change and world food supply. Oxford : Oxford University Press.

SCHLENKER W. et ROBERTS M. [2009], « Nonlinear temperature effects indicate severe damages to U.S. crop yields under climate change », Proceedings of the National Academy of Sciences 106(37): 15594-15598.

SP/CONAGESE [2001], Convention cadre des nations unies sur les changements climatiques. Communication du Burkina Faso, SP/CONAGESE, Ouagadougou, 126p.

Sultan B., Roudier P., Quirion P., Alhassane A., Muller B., Dingkuhn M. et Baron C. [2013], «Assessing climate change impacts on sorghum and millet yields in the Sudanian and Sahelian savannas of West Africa ». Environmental Research Letters, 8(1), 014040.

TigchelaAR M., Battisti D. S., NAYlor R. L. et Ray D. K. [2018], «Future warming increases probability of globally synchronized maize production shocks ». Proceedings of the National Academy of Sciences, 115(26), 6644-6649.

VAITKEVIČIŪTĖ J. [2018], Econometric analysis of the impacts of climate change on agriculture and implications for adaptation. Thèse de doctorat, Université Paris Saclay

Vaitkeviciute J., ChakiR R. et VAN Passel S. [2019], «Climate variable choice in Ricardian studies of European agriculture ». La Revue Economique 70(3): 375-401

White H. [1980], «A heteroskedasticity-Consistent Covariance Matrix Estimator and a Direct Test for Heteroskedasticity », Econometrica 48, 817-838

Wourtese F. et TAYLOR J. [2008], «Migration Income Diversification: Evidence from Burkina Faso ». World Development Vol.36, No. 4, pp. 625-640.

Wright P. G. [1928], The Tariff on Animal and Vegetable Oils. New York: MacMillan. 


\section{Annexe 1. Liste des provinces correspondant aux abscisses de la figure 3}

\section{Partie gauche}

1.Leraba ; 2. Comoe ; 3. Poni ; 4. Kenedougou ; 5. Houet ; 6. Bougouriba ; 7. Noumbiel 8. Tuy ; 9. Sissili ; 10. Ioba ; 11. Koulpelgo 12. Bale 13. Boulgou 14. Nahouri ; 15. Kompienga ; 16. Banwa ; 17. Ziro ; 18. Bazega 19. Mouhoun 20. Kouritenga ; 21. Zoundweogo ; 22. Sanguie ; 23. Gourma ; 24. Boulkiemde ; 25. Ganzourgou ; 26. Kadiogo ; 27. Passore ; 28. Nayala ; 29. Kossi ; 30. Tapoa ; 31. Gnagna ; 32. Kourweogo ; 33. Oubritenga ; 34. Zondoma ; 35. Komandjori 36. Sourou ; 37. Bam ; 38. Sanmatenga ; 39. Namentenga ;40. Loroum ; 41. Yatenga ; 42. Yagha ; 43. Seno ; 44. Soum ; 45. Oudalan

\section{Partie droite}

1.Oudalan; 2. Soum ; 3. Seno ; 4. Yagha ; 5. Namentenga ; 6. Sanmatenga ; 7. Loroum ; 8.Yatenga ; 9.Gnagna ; 10.Komandjori ; 11.Oubritenga ; 12.Bam ; 13. Kourweogo ; 14. Zondoma ; 15.Gourma ; 16.Tapoa ; 17.Ganzourgou ; 18.Kadiogo ; 19. Kouritenga ; 20. Sourou ; 21. Passore ; 22. Boulkiemde ; 23.Kossi ; 24. Nayala ; 25.Kompienga ; 26.Sanguie ; 27.Koulpelgo ; 28.Mouhoun ; 29.Banwa ; 30.Ziro ; 31.Zoundweogo ; 32.Bale ; 33.Noumbiel ; 34.Bazega ; 35.Tuy ; 36.Ioba 37.Bougouriba ; 38.Poni ; 39.Houet ; 40.Sissili ; 41.Kenedougou ; 42.Boulgou ; 43.Nahouri ; 44. Comoe ; 45. Leraba 
Annexe 2. Résultats supplémentaires par céréale

Tableau A1. Résultats des estimations du rendement pour le maïs

\begin{tabular}{|c|c|c|c|c|c|c|c|c|}
\hline $\begin{array}{l}\text { Modèle } \rightarrow \\
\text { Variable } \downarrow\end{array}$ & $\mathbf{R E}$ & & RE_TT & & $\mathbf{F E}$ & & FE_TT & \\
\hline Pluie mai & 2.477 & & 3.242 & & 1.674 & & 2.435 & \\
\hline Pluie mai² & -0.002 & & -0.006 & & 0.000 & & -0.004 & \\
\hline Pluie juin & 7.655 & $* *$ & 6.878 & $* *$ & 6.886 & $*$ & 6.096 & $*$ \\
\hline Pluie juin ${ }^{2}$ & -0.026 & $* *$ & -0.022 & $*$ & -0.025 & $*$ & -0.021 & $*$ \\
\hline Pluie août & 4.786 & $* *$ & 4.416 & $* *$ & 5.073 & $* *$ & 4.634 & $* *$ \\
\hline Pluie août ${ }^{2}$ & -0.010 & $* *$ & -0.010 & $* *$ & -0.011 & $* *$ & -0.010 & $* *$ \\
\hline $\begin{array}{l}\text { Pluie } \\
\text { septembre }\end{array}$ & 7.848 & $* * *$ & 7.609 & $* * *$ & 8.015 & $* * *$ & 7.726 & $* * *$ \\
\hline $\begin{array}{l}\text { Pluie } \\
\text { septembre }\end{array}$ & -0.018 & $* * *$ & -0.018 & $* * *$ & -0.019 & $* * *$ & -0.018 & $* * *$ \\
\hline Année & & & 12.016 & & & & 12.223 & \\
\hline Constante & -583.534 & $*$ & -566.023 & $*$ & -505.950 & & -481.748 & \\
\hline $\begin{array}{l}\text { Effets fixes } \\
\text { par province }\end{array}$ & Non & & Non & & Oui & & Oui & \\
\hline $\mathrm{N}$ & 499 & & 499 & & 499 & & 499 & \\
\hline $\mathrm{R}^{2}$ within & 0.116 & & 0.129 & & 0.118 & & 0.131 & \\
\hline $\mathrm{R}^{2}$ between & 0.362 & & 0.368 & & 0.317 & & 0.328 & \\
\hline $\mathrm{R}^{2}$ overall & 0.208 & & 0.213 & & 0.192 & & 0.198 & \\
\hline
\end{tabular}

$* \mathrm{p}<.05 ; * * \mathrm{p}<.01 ; * * * \mathrm{p}<.001$

RE : modèle à effets aléatoires. RE_TT : avec tendance temporelle.

FE : modèle à effets fixes par province. FE_TT : avec tendance temporelle 
Tableau A2. Résultats des estimations du rendement pour le mil

\begin{tabular}{|c|c|c|c|c|c|c|c|c|}
\hline $\begin{array}{l}\text { Modèle } \rightarrow \\
\text { Variable } \downarrow\end{array}$ & $\mathbf{R E}$ & & RE_TT & & FE & & FE_TT & \\
\hline Pluie mai & -0.590 & & -0.588 & & -0.802 & & -0.906 & \\
\hline Pluie mai² & 0.013 & & 0.013 & & 0.012 & & 0.013 & \\
\hline Pluie juin & 4.121 & $* *$ & 4.156 & $*$ & 4.144 & $* *$ & 4.227 & $* *$ \\
\hline Pluie juin ${ }^{2}$ & -0.015 & & -0.015 & & -0.017 & $*$ & -0.017 & $*$ \\
\hline Pluie août & 1.323 & & 1.328 & & 1.426 & & 1.491 & \\
\hline Pluie août ${ }^{2}$ & -0.003 & & -0.003 & & -0.003 & & -0.003 & \\
\hline Pluie septembre & 3.182 & $*$ & 3.190 & $*$ & 3.210 & $* *$ & 3.241 & $* *$ \\
\hline $\begin{array}{l}\text { Pluie } \\
\text { septembre }\end{array}$ & -0.008 & $*$ & -0.008 & $*$ & -0.008 & $*$ & -0.008 & $*$ \\
\hline Année & & & -0.653 & & & & -1.371 & \\
\hline Constante & 228.193 & & 220.943 & & 243.158 & & 239.619 & \\
\hline $\begin{array}{l}\text { Effets fixes par } \\
\text { province }\end{array}$ & Non & & Non & & Oui & & Oui & \\
\hline $\mathrm{N}$ & 548 & & 548 & & 548 & & 548 & \\
\hline $\mathrm{R}^{2}$ within & 0.067 & & 0.066 & & 0.070 & & 0.070 & \\
\hline $\mathrm{R}^{2}$ between & 0.300 & & 0.308 & & 0.224 & & 0.212 & \\
\hline $\mathrm{R}^{2}$ overall & 0.172 & & 0.175 & & 0.150 & & 0.148 & \\
\hline
\end{tabular}

$* \mathrm{p}<.05 ; * * \mathrm{p}<.01 ; * * * \mathrm{p}<.001$

RE : modèle à effets aléatoires. RE_TT : avec tendance temporelle.

FE : modèle à effets fixes par province. FE_TT : avec tendance temporelle 
Tableau A3. Résultats des estimations du rendement pour le sorgho

\begin{tabular}{|c|c|c|c|c|c|c|c|c|}
\hline $\begin{array}{c}\text { Modèle } \rightarrow \\
\text { Variable } \downarrow\end{array}$ & RE & & RE_TT & & FE & & FE_TT & \\
\hline Pluie mai & 2.739 & $* *$ & 2.864 & $* *$ & 2.242 & $*$ & 2.410 & $*$ \\
\hline Pluie mai² & -0.013 & $*$ & -0.014 & $*$ & -0.012 & & -0.013 & $*$ \\
\hline Pluie juin & 2.509 & & 2.416 & & 2.180 & & 2.041 & \\
\hline Pluie juin ${ }^{2}$ & -0.008 & & -0.007 & & -0.008 & & -0.007 & \\
\hline Pluie août & 2.238 & $*$ & 2.170 & & 2.144 & & 2.046 & \\
\hline Pluie août ${ }^{2}$ & -0.004 & $*$ & -0.004 & & -0.004 & & -0.004 & \\
\hline $\begin{array}{l}\text { Pluie } \\
\text { septembre }\end{array}$ & 4.011 & $* * *$ & 3.982 & $* * *$ & 4.049 & $* * *$ & 3.996 & $* * *$ \\
\hline $\begin{array}{l}\text { Pluie } \\
\text { septembre }^{2}\end{array}$ & -0.010 & $* * *$ & -0.010 & $* * *$ & -0.010 & $* * *$ & -0.010 & $* * *$ \\
\hline Année & & & 1.593 & & & & 2.137 & \\
\hline Constante & 148.172 & & 151.244 & & 219.340 & & 225.148 & \\
\hline $\begin{array}{l}\text { Effets fixes } \\
\text { par province }\end{array}$ & Non & & Non & & Oui & & Oui & \\
\hline $\mathrm{N}$ & 555 & & 555 & & 555 & & 555 & \\
\hline $\mathrm{R}^{2}$ within & 0.070 & & 0.071 & & 0.073 & & 0.074 & \\
\hline $\mathrm{R}^{2}$ between & 0.361 & & 0.360 & & 0.308 & & 0.305 & \\
\hline $\mathrm{R}^{2}$ overall & 0.188 & & 0.188 & & 0.167 & & 0.167 & \\
\hline
\end{tabular}

$* \mathrm{p}<.05 ; * * \mathrm{p}<.01 ; * * * \mathrm{p}<.001$

RE : modèle à effets aléatoires. RE_TT : avec tendance temporelle.

FE : modèle à effets fixes par province. FE_TT : avec tendance temporelle 
Tableau A4. Résultats des estimations du prix pour le maïs

\begin{tabular}{|c|c|c|c|c|c|c|c|c|}
\hline $\begin{array}{l}\text { Modèle } \rightarrow \\
\text { Variable } \downarrow\end{array}$ & RE & & RE_TT & & FE & & FE_TT & \\
\hline $\begin{array}{l}\text { Rendement en t- } \\
1\end{array}$ & -0.037 & $* * *$ & -0.037 & $* * *$ & -0.025 & $* *$ & -0.024 & $* *$ \\
\hline $\begin{array}{l}\text { Prix mondial du } \\
\text { maïs }\end{array}$ & 0.174 & $* * *$ & 0.294 & $* * *$ & 0.180 & $* * *$ & 0.307 & $* * *$ \\
\hline $\begin{array}{l}\text { Indice des prix à } \\
\text { la } \\
\text { consommation }\end{array}$ & 0.991 & $* * *$ & & & 0.906 & $* * *$ & & \\
\hline Année & & & 0.578 & & & & 0.135 & \\
\hline Constante & 54.020 & $* * *$ & 124.928 & $* * *$ & 48.711 & $* * *$ & 113.021 & $* * *$ \\
\hline $\begin{array}{l}\text { Effets fixes par } \\
\text { province }\end{array}$ & Non & & Non & & Oui & & Oui & \\
\hline $\mathrm{N}$ & 446 & & 446 & & 446 & & 446 & \\
\hline $\mathrm{R}^{2}$ within & 0.362 & & 0.327 & & 0.367 & & 0.332 & \\
\hline $\mathrm{R}^{2}$ between & 0.714 & & 0.676 & & 0.706 & & 0.707 & \\
\hline $\mathrm{R}^{2}$ overall & 0.441 & & 0.409 & & 0.423 & & 0.390 & \\
\hline
\end{tabular}

$* \mathrm{p}<.05 ; * * \mathrm{p}<.01 ; * * * \mathrm{p}<.001$. L'intervalle de confiance à $95 \%$ est indiqué entre parenthèses pour la variable d'intérêt (le rendement en t-1).

RE : modèle à effets aléatoires. RE_TT : avec tendance temporelle.

FE : modèle à effets fixes par province. FE_TT : avec tendance temporelle 
Tableau A5. Résultats des estimations du prix pour le mil

\begin{tabular}{|c|c|c|c|c|c|c|c|c|}
\hline $\begin{array}{l}\text { Modèle } \rightarrow \\
\text { Variable } \downarrow\end{array}$ & $\mathbf{R E}$ & & RE_TT & & FE & & FE_TT & \\
\hline $\begin{array}{r}\text { Rendement en t- } \\
1\end{array}$ & -0.019 & $* *$ & -0.020 & $* *$ & -0.026 & $* *$ & -0.028 & $* *$ \\
\hline $\begin{array}{r}\text { Prix mondial du } \\
\text { maïs }\end{array}$ & 0.268 & $* * *$ & 0.357 & $* * *$ & 0.270 & $* * *$ & 0.355 & $* * *$ \\
\hline $\begin{array}{l}\text { Indice des prix à } \\
\text { la consommation }\end{array}$ & 1.555 & $* * *$ & & & 1.498 & $* * *$ & & \\
\hline Année & & & 2.614 & $* * *$ & & & 2.496 & $* * *$ \\
\hline Constante & -7.983 & & 105.960 & $* * *$ & 3.982 & & 114.864 & $* * *$ \\
\hline $\begin{array}{r}\text { Effets fixes par } \\
\text { province }\end{array}$ & Non & & Non & & Oui & & Oui & \\
\hline $\mathrm{N}$ & 502 & & 502 & & 502 & & 502 & \\
\hline $\mathrm{R}^{2}$ within & 0.489 & & 0.457 & & 0.490 & & 0.459 & \\
\hline $\mathrm{R}^{2}$ between & 0.464 & & 0.441 & & 0.408 & & 0.369 & \\
\hline $\mathrm{R}^{2}$ overall & 0.439 & & 0.409 & & 0.429 & & 0.397 & \\
\hline
\end{tabular}

$* \mathrm{p}<.05 ; * * \mathrm{p}<.01 ; * * * \mathrm{p}<.001$. L'intervalle de confiance à $95 \%$ est indiqué entre parenthèses pour la variable d'intérêt (le rendement en t-1).

RE : modèle à effets aléatoires. RE_TT : avec tendance temporelle.

$\mathrm{FE}$ : modèle à effets fixes par province. FE_TT : avec tendance temporelle 
Tableau A6. Résultats des estimations du prix pour le sorgho

\begin{tabular}{|r|c|c|c|c|c|c|c|c|}
\hline $\begin{array}{r}\text { Modèle } \rightarrow \\
\text { Variable } \downarrow\end{array}$ & RE & RE_TT & & FE & & FE_TT & \\
\hline $\begin{array}{r}\text { Rendement en t- } \\
1\end{array}$ & -0.027 & $* * *$ & -0.027 & $* * *$ & -0.027 & $* * *$ & -0.027 & $* * *$ \\
\hline $\begin{array}{r}\text { Prix mondial du } \\
\text { maïs }\end{array}$ & 0.174 & $* * *$ & 0.262 & $* * *$ & 0.177 & $* * *$ & 0.265 & $* * *$ \\
\hline $\begin{array}{r}\text { Indice des prix à } \\
\text { la } \\
\text { consommation }\end{array}$ & 1.056 & $* * *$ & & & 1.001 & $* * *$ & & \\
\hline Année & & & 1.271 & $* * *$ & & & 1.102 & $* *$ \\
\hline Constante & 40.024 & $* * *$ & 116.962 & $* * *$ & 44.706 & $* *$ & 117.791 & $* * *$ \\
\hline $\begin{array}{r}\text { Effets fixes par } \\
\text { province }\end{array}$ & Non & & Non & & Oui & & 0.10 & \\
\hline $\mathrm{N}$ & 501 & & 501 & & 501 & & 501 & \\
\hline $\mathrm{R}^{2}$ within & 0.342 & & 0.312 & & 0.342 & & 0.312 & \\
\hline $\mathrm{R}^{2}$ between & 0.477 & & 0.450 & & 0.473 & & 0.444 & \\
\hline $\mathrm{R}^{2}$ overall & 0.336 & & 0.309 & & 0.335 & & 0.309 & \\
\hline
\end{tabular}

$* \mathrm{p}<.05 ; * * \mathrm{p}<.01 ; * * \mathrm{p}<.001$. L'intervalle de confiance à $95 \%$ est indiqué entre parenthèses pour la variable d'intérêt (le rendement en t-1).

RE : modèle à effets aléatoires. RE_TT : avec tendance temporelle.

FE : modèle à effets fixes par province. FE_TT : avec tendance temporelle 
Tableau A7. Variation du prix en fonction de la pluie, par céréale

\begin{tabular}{|c|c|c|c|c|c|}
\hline & 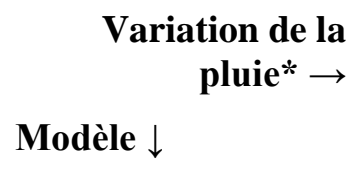 & $-20 \%$ & $-15 \%$ & $-10 \%$ & $-5 \%$ \\
\hline \multirow[t]{4}{*}{ Maïs } & $\mathbf{R E}$ & $8.0 \%$ & $6.0 \%$ & $4.0 \%$ & $2.0 \%$ \\
\hline & RE_TT & $7.9 \%$ & $5.9 \%$ & $4.0 \%$ & $2.0 \%$ \\
\hline & $\mathbf{F E}$ & $5.2 \%$ & $3.9 \%$ & $2.6 \%$ & $1.3 \%$ \\
\hline & FE_TT & $5.0 \%$ & $3.7 \%$ & $2.5 \%$ & $1.2 \%$ \\
\hline \multirow[t]{4}{*}{ Mil } & $\mathbf{R E}$ & $2.1 \%$ & $1.6 \%$ & $1.1 \%$ & $0.5 \%$ \\
\hline & RE_TT & $2.2 \%$ & $1.7 \%$ & $1.1 \%$ & $0.6 \%$ \\
\hline & $\mathbf{F E}$ & $2.9 \%$ & $2.2 \%$ & $1.5 \%$ & $0.7 \%$ \\
\hline & FE_TT & $3.1 \%$ & $2.3 \%$ & $1.6 \%$ & $0.8 \%$ \\
\hline \multirow[t]{4}{*}{ Sorgho } & $\mathbf{R E}$ & $4.0 \%$ & $3.0 \%$ & $2.0 \%$ & $1.0 \%$ \\
\hline & RE_TT & $4.0 \%$ & $3.0 \%$ & $2.0 \%$ & $1.0 \%$ \\
\hline & $\mathbf{F E}$ & $4.0 \%$ & $3.0 \%$ & $2.0 \%$ & $1.0 \%$ \\
\hline & FE_TT & $4.0 \%$ & $3.0 \%$ & $2.0 \%$ & $1.0 \%$ \\
\hline
\end{tabular}

* Variation de la pluie par rapport à la moyenne 2001-2014, pour chaque province, d'un même pourcentage pour les mois de mai, juin, août et septembre.

RE : modèle à effets aléatoires. RE_TT : avec tendance temporelle.

FE : modèle à effets fixes par province. FE_TT : avec tendance temporelle 
Tableau A8. Biais de prix en pourcentage du revenu agricole, par céréale

\begin{tabular}{|c|c|c|c|c|c|}
\hline & 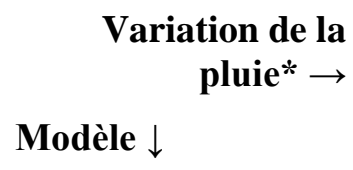 & $-20 \%$ & $-15 \%$ & $-10 \%$ & $-5 \%$ \\
\hline \multirow[t]{4}{*}{ Maïs } & $\mathbf{R E}$ & $109 \%$ & $119 \%$ & $130 \%$ & $142 \%$ \\
\hline & RE_TT & $109 \%$ & $119 \%$ & $130 \%$ & $142 \%$ \\
\hline & $\mathbf{F E}$ & $63 \%$ & $67 \%$ & $71 \%$ & $76 \%$ \\
\hline & FE_TT & $60 \%$ & $63 \%$ & $67 \%$ & $71 \%$ \\
\hline \multirow[t]{4}{*}{ Mil } & $\mathbf{R E}$ & $23 \%$ & $24 \%$ & $25 \%$ & $25 \%$ \\
\hline & RE_TT & $24 \%$ & $25 \%$ & $26 \%$ & $26 \%$ \\
\hline & $\mathbf{F E}$ & $33 \%$ & $34 \%$ & $35 \%$ & $36 \%$ \\
\hline & FE_TT & $36 \%$ & $37 \%$ & $38 \%$ & $39 \%$ \\
\hline \multirow[t]{4}{*}{ Sorgho } & $\mathbf{R E}$ & $49 \%$ & $50 \%$ & $52 \%$ & $54 \%$ \\
\hline & RE_TT & $49 \%$ & $51 \%$ & $53 \%$ & $55 \%$ \\
\hline & $\mathbf{F E}$ & $49 \%$ & $51 \%$ & $52 \%$ & $54 \%$ \\
\hline & FE_TT & $49 \%$ & $51 \%$ & $53 \%$ & $54 \%$ \\
\hline
\end{tabular}

* Variation de la pluie par rapport à la moyenne 2001-2014, pour chaque province, d'un même pourcentage pour les mois de mai, juin, août et septembre.

RE : modèle à effets aléatoires. RE_TT : avec tendance temporelle.

FE : modèle à effets fixes par province. FE_TT : avec tendance temporelle. 


\section{Annexe 3. Résultats des estimations en fonction des pluies et des températures}

Lorsque les températures sont incluses en plus des pluies parmi les variables explicatives du rendement, ces variables ne sont pas statistiquement significatives à $5 \%$ pour les mois de mai, juillet et octobre. Aussi, nous montrons les résultats incluant comme variables explicatives les pluies et températures des mois de juin, août et septembre (Tableau A9). Pour les pluies, on obtient les relations concaves attendues et les valeurs des coefficients sont très proches de celles des régressions sans les températures (Tableau 2) pour juin et septembre. Le coefficient de la tendance temporelle, dans les modèles où elle est présente, est similaire. La valeur du coefficient de la pluie en août est plus faible, et celui de la température n'est pas significatif au seuil de 5\% pour juin. Le signe des coefficients de la température est opposé entre août et septembre : on obtient une relation concave pour août et convexe pour septembre - mais dans les deux cas, la moyenne se trouve dans la partie décroissante de la courbe.

D'un point de vue agronomique, la logique de ces relations n'est pas claire, aussi nous craignons qu'elles ne soient affectées par la forte corrélation entre pluie et températures, mentionnée en section 2.4. C'est pourquoi, dans la section 4, nous présentons les résultats concernant le biais de prix sur la base des modèles où les températures n'interviennent pas. Néanmoins, nos conclusions sur le biais de prix restent qualitativement valides et quantitativement proches en calculant le biais de prix sur la base de modèles où les températures figurent également parmi les déterminants des rendements.

Dans le Tableau A10, nous calculons l'effet sur le prix des pluies et des températures sur le rendement, sur la base des coefficients présentés au Tableau A9. Le rendement augmente avec la pluie et diminue avec la température, de manière cohérente avec la plupart des travaux portant sur la zone soudano-sahélienne (Roudier et al., 2011). L'effet d'une hausse des températures, même limitée à $1^{\circ} \mathrm{C}$ (par rapport à la moyenne 2001-2014) est nettement supérieur à celui d'une baisse des pluies de 20\%. Sans changement de température, l'effet de la pluie est plus faible que celui obtenu par les régressions sans les températures, comme on peut le voir en comparant la dernière colonne du Tableau A9 avec les quatre premières lignes du Tableau 4. 
Tableau A9. Résultats des estimations du rendement de l'ensemble des trois céréales en fonction des pluies et des températures

\begin{tabular}{|c|c|c|c|c|c|c|c|c|}
\hline $\begin{array}{c}\text { Modèle } \rightarrow \\
\text { Variable } \downarrow\end{array}$ & RE & & RE_TT & & FE & & FE_TT & \\
\hline Pluie juin & 4.19 & $*$ & 3.05 & & 3.92 & $* *$ & 2.85 & $*$ \\
\hline Pluie juin ${ }^{2}$ & -0.02 & $*$ & -0.01 & & -0.02 & $*$ & -0.01 & * \\
\hline Pluie août & 1.24 & & 0.78 & & 1.48 & $*$ & 1.03 & \\
\hline Pluie août ${ }^{2}$ & -0.00 & $* *$ & -0.00 & & -0.00 & * & -0.00 & \\
\hline $\begin{array}{l}\text { Pluie } \\
\text { septembre }\end{array}$ & 4.01 & $* * *$ & 3.86 & $* * *$ & 4.29 & $* * *$ & 4.14 & $* * *$ \\
\hline $\begin{array}{l}\text { Pluie } \\
\text { septembre }\end{array}$ & -0.01 & $* * *$ & -0.01 & $* * *$ & -0.01 & $* * *$ & -0.01 & $* * *$ \\
\hline $\begin{array}{l}\text { Température } \\
\text { juin }\end{array}$ & 114.82 & & 116.32 & & 467.68 & & 448.77 & \\
\hline $\begin{array}{l}\text { Température } \\
\text { juin }^{2}\end{array}$ & -2.35 & & -2.86 & & -7.74 & & -7.90 & \\
\hline $\begin{array}{l}\text { Température } \\
\text { août }\end{array}$ & 1761.68 & $*$ & 1971.22 & $* *$ & 2929.91 & $* *$ & 3080.01 & $* *$ \\
\hline $\begin{array}{l}\text { Température } \\
\text { août }{ }^{2}\end{array}$ & -33.38 & $*$ & -37.01 & $* *$ & -54.45 & $* *$ & -57.00 & $* *$ \\
\hline $\begin{array}{l}\text { Température } \\
\text { sept. }\end{array}$ & -3436.19 & $* * *$ & -3265.73 & $* * *$ & -2771.77 & $* * *$ & -2649.69 & $* * *$ \\
\hline $\begin{array}{l}\text { Température } \\
\text { sept. }\end{array}$ & 59.88 & $* * *$ & 57.10 & $* * *$ & 48.40 & $* * *$ & 46.47 & $* * *$ \\
\hline Année & & & 9.49 & $* *$ & & & 8.89 & $*$ \\
\hline Constante & 24974.67 & $*$ & 19868.85 & & -6526.91 & & -9834.41 & \\
\hline $\begin{array}{l}\text { Effets fixes } \\
\text { province }\end{array}$ & Non & & Non & & Oui & & Oui & \\
\hline $\mathrm{N}$ & 551 & & 551 & & 551 & & 551 & \\
\hline $\mathrm{R}^{2}$ within & 0.13 & & 0.15 & & 0.15 & & 0.17 & \\
\hline $\mathrm{R}^{2}$ between & 0.65 & & 0.66 & & 0.48 & & 0.53 & \\
\hline $\mathrm{R}^{2}$ overall & 0.48 & & 0.49 & & 0.33 & & 0.36 & \\
\hline
\end{tabular}

$* \mathrm{p}<.05 ; * * \mathrm{p}<.01 ; * * * \mathrm{p}<.001$

RE : modèle à effets aléatoires. RE_TT : avec tendance temporelle.

FE : modèle à effets fixes par province. FE_TT : avec tendance temporelle 
Tableau A10. Variation du rendement en fonction de la pluie et de la température

\begin{tabular}{|c|c|c|c|c|c|}
\hline $\begin{array}{l}\text { Variation de la } \\
\text { pluie* }\end{array}$ & 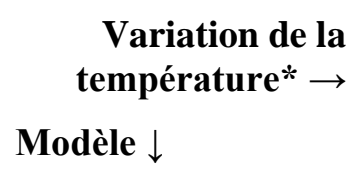 & $+3^{\circ} \mathrm{C}$ & $+2^{\circ} \mathrm{C}$ & $+1^{\circ} \mathrm{C}$ & $+0^{\circ} \mathrm{C}$ \\
\hline \multirow[t]{4}{*}{$-20 \%$} & RE & $-48 \%$ & $-42 \%$ & $-29 \%$ & $-13 \%$ \\
\hline & RE_TT & $-50 \%$ & $-44 \%$ & $-27 \%$ & $-10 \%$ \\
\hline & FE & $-47 \%$ & $-40 \%$ & $-22 \%$ & $-14 \%$ \\
\hline & FE_TT & $-50 \%$ & $-44 \%$ & $-21 \%$ & $-12 \%$ \\
\hline \multirow[t]{4}{*}{$-10 \%$} & $\mathbf{R E}$ & $-42 \%$ & $-41 \%$ & $-23 \%$ & $-7 \%$ \\
\hline & RE_TT & $-44 \%$ & $-40 \%$ & $-21 \%$ & $-5 \%$ \\
\hline & $\mathbf{F E}$ & $-40 \%$ & $-33 \%$ & $-15 \%$ & $-7 \%$ \\
\hline & FE_TT & $-44 \%$ & $-34 \%$ & $-15 \%$ & $-6 \%$ \\
\hline \multirow[t]{4}{*}{$0 \%$} & $\mathbf{R E}$ & $-35 \%$ & $-28 \%$ & $-16 \%$ & $0 \%$ \\
\hline & RE_TT & $-39 \%$ & $-29 \%$ & $-16 \%$ & $0 \%$ \\
\hline & FE & $-33 \%$ & $-19 \%$ & $-8 \%$ & $0 \%$ \\
\hline & FE_TT & $-38 \%$ & $-22 \%$ & $-9 \%$ & $0 \%$ \\
\hline
\end{tabular}

* Variation par rapport à la moyenne 2001-2014, pour chaque province, d'un même pourcentage (pluie) ou d'un même montant (température) pour les mois de mai, juin, août et septembre.

RE : modèle à effets aléatoires. RE_TT : avec tendance temporelle.

FE : modèle à effets fixes par province. FE_TT : avec tendance temporelle. 


\section{Annexe 4. Variations du bien-être, de l'utilité du consommateur et du coût de}

production.

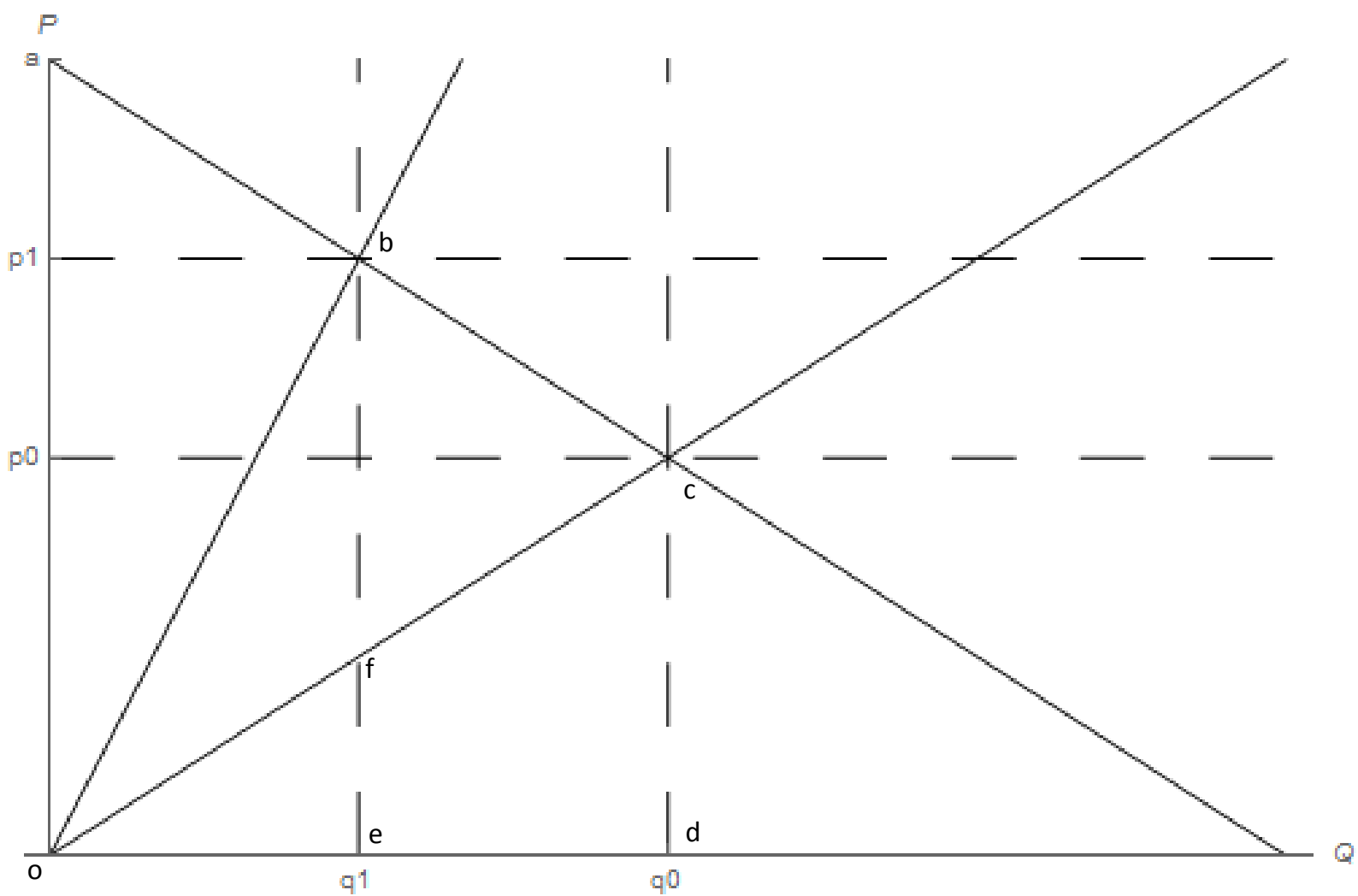

Le bien-être est égal par définition à la différence entre l'utilité pour le consommateur et le coût de production.

L'utilité du consommateur est égal à la surface (o,a,c,d) à l'équilibre $\left(p_{0}, q_{0}\right)$, et à la surface $(\mathrm{o}, \mathrm{a}, \mathrm{b}, \mathrm{e})$ à l'équilibre $\left(p_{1}, q_{1}\right)$. La différence est donc la surface $(\mathrm{c}, \mathrm{d}, \mathrm{b}, \mathrm{e})$, notée $(\mathrm{B}+\mathrm{C})$ sur la Figure 1.

Le coût de production est égal à la surface $(\mathrm{o}, \mathrm{c}, \mathrm{d})$ à l'équilibre $\left(p_{0}, q_{0}\right)$, et à la surface $(\mathrm{o}, \mathrm{b}, \mathrm{e})$ à l'équilibre $\left(p_{1}, q_{1}\right)$. La différence est donc la surface $(\mathrm{o}, \mathrm{f}, \mathrm{b})-(\mathrm{c}, \mathrm{d}, \mathrm{e}, \mathrm{f})$ notée $(\mathrm{D}+\mathrm{E}-\mathrm{F})$ sur la Figure 1. 